1 A ferrofluid-based sensor to measure bottom shear stresses under currents

2 and waves

3 ROSARIA E. MUSUMECI, Assistant Professor, Department Civil Engineering and

4 Architecture, University of Catania, Catania, Italy

5 Email: rmusume@dica.unict.it (author for correspondence)

6 VINCENZO MARLETTA, Post-doc, Department of Electric, Electronics and Computer

7 Engineering, University of Catania, Catania, Italy

$8 \quad$ Email: vincenzo.marletta@dieei.unict.it

9 AGUSTIN SANCHEZ-ARCILLA, Full Professor, Laboratori de Enginyeria Maritima,

10 Technical University of Catalunya, Barcelona, Spain

11 Email: agustin.arcilla@upc.edu

12 ENRICO FOTI, Full Professor, Department Civil Engineering and Architecture, University of 13 Catania, Catania, Italy

14 Email:efoti@dica.unict.it

15

16

17 Running Head: Ferrofluid sensor to measure bottom shear stresses.

18 


\title{
1 A ferrofluid-based sensor to measure bottom shear stresses under currents

\begin{abstract}
The measurement of the near-bottom flow characteristics is crucial to correctly understand coastal processes. Aiming at overcoming some of the limits of present state-of-the-art measuring instruments, we propose a novel approach to measure bottom shear stress under currents and waves based on the exploitation of magneto-rheological fluids, named ferrofluids. In particular the deformation of a magnetically controlled ferrofluid drop $\mathrm{O}(0.01 \mathrm{ml})$ is transduced by a conditioning circuit into an output voltage which is proportional to the bottom shear stress. Calibration curves are presented for both steady-current and regular wave conditions, over fixed and weakly mobile beds, by showing that the behaviour of the proposed measuring system can be assumed linear. In the range of the investigated parameters, it is shown that the working range is comprised between $0.08 \mathrm{~N} / \mathrm{m}^{2}$ and an upper limit which is a function of the controlling magnetic field and of the flow type.
\end{abstract}

Keywords: magneto-rheological fluids, bottom shear stress measurement, gravity waves, transducer, Rosensweig effect

\section{Introduction}

The measure of shear stresses close to solid boundaries is crucial in several fields, ranging from hydraulics to aerospace. The interaction between flow and solid walls influences to a great extent the characteristics of the flow itself. In hydraulics, the knowledge of wall shear stresses is fundamental to evaluate energy dissipation of the flow and to assess the flow interaction with the bottom. This is extremely relevant not only to optimize the design of pipelines or open-channels, but also to investigate and quantify sediment transport processes in rivers, estuaries or along the coasts. In other research fields, such as automotive, naval or aerospace industries, the correct evaluation of wall shear stresses is needed to design solutions, shapes and materials which allow to reduce energy losses and in turn fuel consumption.

In the past, bed shear stresses have been measured by means of simple instruments, such as Preston tubes and Stanton tubes (Head and Rechenberg, 1962). Nowadays, bottom shear stresses $\tau_{0}$ are still indirectly derived from the logarithmic velocity profile or integral momentum method, by using acoustic sensors, such as Acoustic Doppler Velocimeter (Musumeci, Cavallaro, Foti, Scandura, and Blondeaux, 2006) or optical sensors, such as Laser Doppler Velocimeter or PIV systems (Cox, Kobayashi, and Okayasu, 1996; Nimmo Smith, Atsavapranee, Katz, and Osborne, 2002). Direct measurements of the bed shear stresses can be performed by using flush mounted shear plates, which integrate the force over 
1 a relatively large area (Riedel, Kamphuis, and Brebner, 1974; Rankin and Hires, 2000; Barnes

2 and Baldock, 2007; You, Yin, and Huo, 2009). In the aerospace community, in the last

3 decade, great attention has been devoted to direct measurements of bed shear stresses by means of Micro-Electro-Mechanical System (MEMS)-based sensors, which exploit the capabilities of silicon micro-machining technology (Löfdhal and Gad-el-Hak, 1999; Rezazadeh, Loftiani, and Khalilarya, 2009). Thermal sensors are also widely used. For example, hot film anemometers relate the intensity of flow or shear to the forced convection of heat from the sensor surface (Gust, 1988; Sumer, Arnskov, Christiansen, and Jorgensen, 1993). Recently, bioluminescent organisms have been used to map shear stress over a rippled seabed (Foti, Faraci, Foti, and Bonanno, 2010). However, difficulties emerged, since dinoflagellates used to sense the shear stresses can survive only under controlled temperatures and salinity of the water and their bioluminescence is emitted at night and consequently experiments had to be carried out during the night, which is not efficient for the facility operation.

For extensive reviews of the available methodologies and instruments used to estimate wall shear stress, the reader is referred to the work of Winter (1977), Ackerman and Hoover (2001), Naughton and Sheplak (2002), Wallace and Vukoslavčević (2010), Bagherimiyab and Lemmin (2013), and Musumeci, Marletta, Andò, Baglio and Foti (2015a). Table 1 summarizes some of the main characteristics of the measuring systems presently used, along with their advantages and disadvantages. It appears that the latters are mainly related to: (i) the capability to obtain point measurements of small bottom shear stress using a robust system; (ii) the fact that several methodologies rely on the validity of the logarithmic profile; (iii) non-uniqueness of the calibration relationship; (iv) sources of error due to misalignments between the flow and the axis of the instrument, and to mechanical or thermal disturbing processes; (v) high fragility of the sensors which cannot be used in the presence, for example, of moving sediments.

Aiming at complementing the present available instrumentations and at obtaining a measurement methodology that can be applied also in the presence of sandy bottoms, over which state-of-the-art-instruments usually fail, in the present work we investigate the performances of an innovative ferrofluid sensor able to measure bottom shear stresses under currents and waves, which exploits the Rosensweig effect (Cowley and Rosensweig, 1967). Preliminary tests of the measuring systems have been performed by Musumeci, Marletta, Andò, Baglio, and Foti (2015b), based on a limited set of steady current experiments in the presence of a smooth fixed bed, and by Musumeci et al. (2015a) in the presence of regular surface waves on a rough bottom covered with medium-coarse sand. However, in the above previous works not only the number of tested flow conditions was 
1 limited, but also neither the effects on the performances of the system of the strength of the

2 external magnetic forces that generate the Rosensweig effect were systematically quantified nor movable beds were considered to investigate possible disturbances of the impacts of moving sediments on the ferrofluid sensor. Moreover, a comparison between the behavior of the system in steady and oscillatory flows was not performed.

The aim of the present contribution is to characterize the behavior of the proposed ferrofluid sensor under both steady currents and regular surface waves. In particular, here, two extensive experimental campaigns, one in steady flow conditions, over a smooth fixed bed, and one under regular surface waves over a coarser and a finer sandy bottoms, are presented. The results allow to define the range of application of the proposed measuring system and to quantitatively assess the errors of the calibration relationship as a function of the sensor setup and of the flow conditions, both in unidirectional flow and in oscillatory flows.

The paper is organized as follows: Section 2 introduces the measurement methodology based on the use of the ferrofluid, the experimental methodology implemented to create the ferrofluid sensor through the Rosensweig effect, and the architecture of the inductive transducer; Section 3 describes the experimental campaign carried out to validate the proposed measuring system in the presence of steady currents and of regular waves; Section 4 presents the experimental results, while in Section 5 the calibration of the proposed methodology is discussed along with an estimate of the measuring errors. The main conclusions along with possible future developments are summarized in Section 6.

\section{Ferrofluid-based measurement methodology}

Ferrofluids are two-state systems made up by solid small ferromagnetic particles (size $1 \div 15 \mathrm{~nm}$ ) dispersed in an organic non-magnetic liquid solvent (Odenbach, 2002). Such materials behave like Newtonian liquid when the applied magnetic field is null or very small. If a critical magnetization is achieved, ferrofluids can experience forces due to magnetic polarization (Odenbach, 2004). At the microscopic scale, long chains of particles are formed in the direction of the magnetic field, whereas at the macro-scale a series of hexagonal pattern deformations aligned along the magnetic field appears (Cowley and Rosensweig, 1967). Such a phenomenon is known in the literature as Rosensweig effect and it disappears as soon as the magnetic field is removed. Figure 1(a) shows an example of such an effect with the formation of hexagonal conical patterns induced on a relatively large amount of ferrofluid $\mathrm{O}(10 \mathrm{ml})$ by an external magnetic field. Figure 1(b) schematically visualizes how such patterns are aligned along the magnetic field line. The application of a magnetic field on a small amount of ferrofluid induces the generation of a single fluidic pattern with a conical shape (see Figure 1c 
and 1d), whose axis is aligned with the direction of the magnetic field, and whose dimensions are very small, since the height of the ferrofluid spike is $\mathrm{O}(1 \mathrm{~mm})$ (see Figure 2).

Being non-miscible with water, the ferrofluid spike acts as a sensor of the bottom shear stresses. Indeed, in hydrostatic conditions the forces acting on the ferrofluid are its own weight, the hydrostatic forces and the external magnetic field. In this condition, the ferrofluid spike remains where it has been generated, i.e. at the measuring station (see Figures 1c and 3a). Besides the gravitational and the magnetic forces, $\mathbf{G}$ and $\mathbf{M}$, under the action of a water flow, the ferrofluid is also subject to dynamic forces, such as the pressure drag $\mathbf{D}$, lift $\mathbf{L}$, friction on the surface of the spike $\mathbf{F}_{\mathrm{s}}$, and drag generated at the bottom of the ferrofluid spike $\mathbf{D}_{\mathbf{b}}$. The order of magnitude of the forces involved in the dynamic equilibrium can be estimated by assuming similarities with other types of flows. In particular, by approximating the volume of the ferrofluid spike to that of a cone with the same diameter and the same height, the weight of the control volume $\mathbf{G}$ is $\mathrm{O}\left(10^{-5} \mathrm{~N}\right)$. The magnetic force $\mathbf{M}$ exerted by the permanent magnets to generate the spike and to keep it in place could not be directly measured at present. However, it turns out that such a force should be large enough to compensate the largest force in the equilibrium, which would be otherwise not equilibrated. The mean form drag force exerted on the spike due to pressure in the flow direction $\mathbf{D}$ should be $\mathrm{O}\left(10^{-7} \div 10^{-4} \mathrm{~N}\right)$, with the drag coefficient $1.5 \div 2.5$, i.e. approximatively equal to that of a cylinder in the same range of Reynolds numbers (Sumer and Fredsøe, 1997). The lift force $\mathbf{L}$, which is orthogonal to the flow direction, should be $\mathrm{O}\left(10^{-7} \div 10^{-4} \mathrm{~N}\right)$, where the lift coefficient has been assumed equal to the mean value of the measurements on rigid cone of Okamoto and Yagita (1977), which is about 0.3. The friction generated on the surface of the ferrofluid spike $\mathbf{F}_{\text {s }}$ should be $\mathrm{O}\left(10^{-7} \div 10^{-4} \mathrm{~N}\right)$, since considering a similarity with the case of smooth and rough cylinders, it has been demonstrated by Achenbach (1968) that $\mathbf{F}_{\mathrm{s}}$ is 2-3\% of $\mathbf{D}$. Concerning $\mathbf{D}_{\mathbf{b}}$, this is the drag generated at the base of the ferrofluid spike by bottom shear stresses. Indeed, since the ferrofluid remains at the liquid state, a circulation at the base of the ferrofluid must be induced at the bottom of the ferrofluid, as a consequence of the continuity of velocity and pressure at the water-ferrofluid interface. Several simplifying hypotheses can be considered to get a rough estimate of the order of magnitude of $\mathbf{D}_{\mathbf{b}}$. First of all, the shear stresses at the bottom of the ferrofluid can be estimated by assuming a Newtonian behavior. Moreover the vertical velocity gradient is assumed similar to that of the outside water, since at the interface the water velocity and the ferrofluid velocity must be the same. Finally considering that the mean value of the dynamic viscosity of the ferrofluid should vary in the

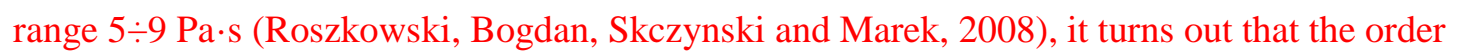
of magnitude of $\mathbf{D}_{\mathbf{b}}$ should be $\mathrm{O}\left(10^{4} \mathrm{~N}\right)$.

Considering the above hypotheses, it appears that the largest force in the equilibrium should be the drag force at the bottom of the ferrofluid spike, mainly because of the very large 
1 dynamic viscosity of the ferrofluid material. All the other forces, except the magnetic force

2 which must compensate $\mathbf{D}_{\mathbf{b}}$, are very small and can be neglected. It turns out that under the

3 flow action, the dynamic equilibrium leads to deformations and displacements of the ferrofluid spike, which are thus proportional to the magnitude of the bottom shear stress.

Figures $3 b$ and $3 c$ qualitatively show how the displacement of the ferrofluid spike increases as the bed shear stress increases. It follows that through an appropriate calibration of the system, shear stress measurements can be obtained by recovering the deformation of the spike. It should be mentioned that the frequency response of the ferrofluid spike to the action of the water flow is $\mathrm{O}(5 \mathrm{~Hz})$ (Andò, Baglio and Beninato, 2012). Therefore the proposed system is suitable to measure Reynolds-averaged flow characteristics and it cannot be used for turbulence measurements.

Andò, Baglio, Trigona, and Faraci (2009) were the first to discuss the possibility to measure the near-bottom velocity profile by qualitatively observing the deformation of a ferrofluid, using a web-cam. In the present work a small drop of ferrofluid, $\mathrm{O}(0.01 \mathrm{ml})$, subject to the Rosensweig effect located at the bed is used to estimate bottom shear stress, by quantitatively measuring the deformation and displacement of the ferrofluid spike induced by the action of the flow through of an inductive readout strategy.

\subsection{Inductive readout strategy}

The transfer of the displacement of the ferrofluid spike's center of mass into a measurable output signal is carried out by means of an on purpose developed inductive readout strategy which makes use of two copper planar coils photoligraphically engraved onto a printed circuit board. The architecture of the inductive transducer which senses the flow-induced has been preliminarily investigated by Musumeci et al. (2015b).

In particular, a PET (PolyEthylene Terephthalate) substrate $100 \mu \mathrm{m}$ thick was used to cover the planar coils in order to electrically isolate the coils from the water and the ferrofluid drop. The ferrofluid drop is located above the two engraved copper coils. Below the flume bottom, one or more permanent magnets are used to generate the external magnetic field, which at the same time produces the ferrofluid spike and maintains it at the measuring station. In order to obtain a single spike shape, an iron nail is used to concentrate the external magnetic field. In the case of movable bottom experiments, to contrast buoyancy or drag effects on the coils, the proposed system can be easily buried within the sand by using a support of the board to increase the weight and to facilitate the burial. Here a Polymethylmethacrylate (PMMA) Perspex ${ }^{\circledR}$ plate about $1 \mathrm{~cm}$ thick, $6 \mathrm{~cm}$ width an $15 \mathrm{~cm}$ long is used.

The ferrofluid spike sits initially in the middle between the two coils. The movement 
1 of the ferrofluid spike, due to the external water flow, produces a variation in the magnetic

2 permeability $\mu$ of the water layer adjoining the two coils and consequently a variation of the

3 inductance $\boldsymbol{L}$ in both the coils. A conditioning circuit is used to transduce the variation of

4 inductance into a direct current (DC) signal, whose value is proportional to the ferrofluid's movement. The system is calibrated in such a way that in hydrostatic conditions the output voltage $V_{\text {out }}$ is null and in dynamic condition a $V_{\text {out }}$ different from zero is obtained, which is proportional to the flow. The design of the conditioning circuit has been carefully addressed in order to reach a stable behaviour of the system. A detailed description of the components of the conditioning circuit was provided in Musumeci et al. (2015a) and in Musumeci et al. (2015b).

\section{Experimental campaign}

The experimental calibration of the system for measuring bed shear stresses under currents and waves has been carried out at the Hydraulic Laboratory of the University of Catania.

In the present experiments, the commercial ferrofluid EFH1 produced by the multinational company Ferrotec (www.ferrotec.com) has been used. Such a ferrofluid is specifically designed for educational purposes, therefore it presents negligible hazards for human health and environment. Moreover, during the experiments, about $80 \%$ of the employed ferrofluid has been retrieved from the experimental setup and reused. The waste of the very tiny amount of ferrofluid, which could not be recycled, has been treated according to the national procedure on disposal of waste oils. Although the exact composition of the material is a proprietary information of Ferrotec, the data sheet provided by the producer reveals that the mixture is made up by magnetite (about $3-15 \%$ of the volume), an oil soluble dispersant (6-30\% of the volume) and a carrier liquid (55-91\% of the volume). The specific gravity is about 1.195 .

The volume of ferrofluid used to generate the ferrofluid spike by Rosensweig effect is equal or smaller than $0.02 \mathrm{ml}$ in all the experiments. Such a volume has been optimized to easily obtain a single ferrofluid spike by using an insulin syringe. Indeed a larger drop would generate more than one spike, due to the action of external magnetic field. On the other hand, it is difficult to produce a smaller drop. To create the Rosensweig effect, several types of permanent magnets with different attraction forces $\boldsymbol{F}$, sold by Supermagnete (www.supermagnete.it), have been used (see Table 2). Since, the dynamic equilibrium of the ferrofluidic sensor is at leading order the result of the balance between the intensity of the external retaining magnetic field and the hydrodynamic forces related to the bottom shear stress, in order to quantitatively assess the effect of the first one on the measurement system, 
various combinations of permanents magnets are considered during the tests.

Preliminary tests have been carried out by Musumeci et al. (2015a) in order to assess the behaviour of the conditioning circuit in hydrostatic conditions. In particular, the system has been used within a tank with water depths which varied in the range $h=0 \div 60 \mathrm{~cm}$. It has been found that the relationship between changes of the hydrostatic water level $\Delta h$ and of the output voltage of the conditioning circuit $\Delta V_{\text {out }}$ is linear. The relationship $\Delta h=-1.81 \Delta V_{\text {out }}$ is independent on the intensity of the external controlling magnetic field and on the presence of the ferrofluid, therefore it is a characteristic of the conditioning circuit.

The experimental calibration of the proposed measurement system has been performed considering two types of flow conditions: steady currents and regular waves.

\subsection{Steady current tests}

In order to produce highly repeatable experimental conditions, the proposed measuring system has been applied in a small scale flume, which is $3.60 \mathrm{~m}$ long, $0.15 \mathrm{~m}$ wide and 0.36 $\mathrm{m}$ high. In the present experiments, the bottom is smooth and the slope is equal to $1.36 \%$. A constant discharge has been recirculated by means of an electro-pump. The value of the discharge can be accurately set by means of a control weir coupled with an electromagnetic flowmeter. In the present tests, subcritical steady flow conditions with a decelerated current are considered.

In order to guarantee that the bottom boundary layer is fully developed, the ferrofluid drop has been located at a distance of $2.57 \mathrm{~m}$ from the flow entrance to the flume. The ferrofluid sits at the center of the printed circuit board, which is glued onto the PMMA flume bottom. The iron nail used to focus the lines of the external controlling magnetic field is inserted in a small hole on the lower external side of the flume bottom itself and the permanent magnets are attached below it.

A $10 \mathrm{MHz}$ Sontek micro-Acoustic Doppler Velocimeter (ADV) has been used to obtain measurements of the mean velocity profiles. The control volume of the ADV is a cylinder 9 $\mathrm{mm}$ high with a volume equal to $0.3 \mathrm{~cm}^{3}$, located $5 \mathrm{~cm}$ far from the transmitter. The timeaveraged velocity profiles are calculated by averaging the $3 \mathrm{D}$ velocity time series measured at least at ten elevations in the range $0.5-5 \mathrm{~cm}$ above the bed. Each time series is 60 seconds long, with a sampling frequency equal to $30 \mathrm{~Hz}$. The water depth $h$ at the measuring station is measured by means of a water level gauge throughout the experiments, being in the range $10.5 \div 11.0 \mathrm{~cm}$ for all the investigated steady current conditions.

Figure 4 shows a sketch of the experimental apparatus and the setup of the ferrofluid sensor and of the printed circuit board with the two planar coils. 
In order to obtain an estimate of the average bottom shear stresses, the ADV's measurements have been best-fitted within the log layer $\left(y^{+}>30\right)$ using the classical wall log law to estimate the friction velocity $u^{*}$ and, in turn, the bottom shear stress $\tau_{x 0}$. Besides the value of $\tau_{x}$, the estimated value of $u^{*}$ allows to determine the average velocity at the ferrofluid location $U_{F F}$, as the theoretical velocity at $y^{+}=y_{F F} \cdot u^{*} / v$, with $y_{F F}=1 \mathrm{~mm}$. A shown in Figure 5, depending on the flow conditions, the ferrofluid tip may be located in in the log layer, in the buffer layer $\left(5<y^{+}<30\right)$ or in the viscous sublayer $\left(y^{+}<5\right)$, where the linear law and the van Driest's profiles respectively hold.

By varying the recirculated water discharge, the velocity $U_{F F}$ varies within the range $0 \div 20 \mathrm{~cm} / \mathrm{s}$. Table 3 reports a summary of the investigated experimental conditions, in terms of water depth $h$, estimated depth-averaged mean velocity in the flow direction $\bar{U}$, velocity at the ferrofluid location $U_{F F}$, bottom shear stress $\tau_{x 0}$ and Reynolds number $R e=U h / v$.

In order to determine the effect of the intensity of the controlling magnetic field on the sensitivity of the sensor and on the repeatability of the measurements, several tests have been carried out in the presence of steady currents by changing the number and types of permanent magnets used to generate the Rosensweig effect on the ferrofluid drop. Some combinations of permanent magnets have been used at least three times to verify the repeatability of the measurements. Table 4 reports an overview of the investigated combinations of permanent magnets used to generate the Rosensweig effect on the ferrofluid drop. An estimate of the overall nominal attraction force $\boldsymbol{F}$ is also shown in the Table. Since it is extremely difficult to measure the effective magnetic field in the presence of water, here, for simplicity sake, it is assumed that $\boldsymbol{F}$ increases linearly with the specific attraction force of each magnet. Such a simplification provides an indication of the magnetic force acting on the ferrofluid. Each one of the combinations reported in Table 4 has been tested by varying the near-bottom velocities from the minimum to the maximum value, i.e. by considering the flow conditions described in Table 3. The experiments performed in still water conditions are used as reference condition (e.g. flow condition $\mathrm{C} 0$ in Table 3).

\subsection{Regular wave tests}

The proposed measurement methodology has been tested in the presence of regular surface waves as well. The experiments have been carried out in a wave tank which is $18 \mathrm{~m}$ long, 3.6 $\mathrm{m}$ wide and $1 \mathrm{~m}$ deep, equipped with an electronically-controlled hydraulic piston-type wavemaker. At the onshore end of the tank a 1:5 planar sloping beach made up by marble stones $\left(d_{50}=3 \mathrm{~cm}\right)$ allows to control wave reflection within the tank.

During wave tests, the printed circuit board with the two copper coils has been glued 
onto a Perspex plate about $1 \mathrm{~cm}$ thick. The plate serves as a support for the iron nail and the permanent magnets. Such a system replicates the setup used for the steady current tests, but in the present case it has been partly buried into a sandy bottom, with the surface of the coils at the level of the initial bed, to test the behaviour of the system in the presence of sediments. Two types of well-sorted quartz sand have been used in the tests, characterized by a median diameter equal to $d_{50}=0.24 \mathrm{~mm}$ and $d_{50}=0.56 \mathrm{~mm}$ respectively. The measuring point is located about $8 \mathrm{~m}$ onshore of the wavemaker, at a section where the wave motion is fully developed. A resistive wave gauge has been used to measure the characteristics of the waves, such as water depth $h$, wave period $T_{\text {wave }}$ and wave height $H$, by adopting a zero-upcrossing method.

Figure 6 shows a planar view of the experimental wave tank along with the setup of the ferrofluid sensor with the printed circuit board on the coarser sandy bottom. It may be noticed that the dimensions of the ferrofluid spike are similar to that of the sediments.

Monochromatic waves have been generated with water depths in the range $h=0.20 \div 0.30 \mathrm{~m}$, wave heights in the range $H=0.06 \div 6.66 \mathrm{~cm}$ and wave periods in the range $T_{\text {wave }}=0.10 \div 2.01 \mathrm{~s}$. The characteristics of the waves have been set to obtain waves both in deep, intermediate and shallow water depths. In particular, deep water tests have been performed to test the behaviour of the conditioning circuit. Indeed, while the ferrofluid sensors is not deformed if there is no flow at the bottom, the conditioning circuit could be in principle be influenced by the varying total water depth. Deep water conditions provide an ideal situation in which the free surface elevation changes, but the bottom shear stress should be null, therefore the ferrofluid deformation should be null and in turn the output voltage should be negligible.

By applying linear wave theory, the wavelength $L_{\text {wave }}$, the amplitude of the potential velocity at the bottom in the wave direction $U_{0}$ and the oscillations amplitude at the bottom $A$ can be estimated. The maximum wave bottom shear stress $\tau_{0 \max }$ is estimated as a function of the wave friction factor $f_{w}=\left.2 \tau_{0 \max }|\rho| U_{0}\right|^{2}$. Since the bottom is rough, the present tests are in the transitional regime, therefore $f$ should be in principle a function of both the Reynolds number $R e_{w}=A U_{0} / v$, where $v$ is the kinematic viscosity, and of the relative roughness $A / k_{N}$, where $k_{N}$ is Nikuradse roughness, which can be estimated as $k_{N}=2.5 d_{50}$. In this regime, in principle the implicit formula proposed by Myrhaug (1989) could be used to estimate $f_{w}$. However, it turns out that in the region of the present Reynolds number $R e_{w}<3000$ and relative roughness $A / k_{N}<36$, the predictions of the Myrhaug's formula collapse in the laminar regime, where $f_{w}=2 / \sqrt{\operatorname{Re}_{w}}$.

In order to investigate the behaviour of the ferrofluid sensor and of the inductive readout measurement strategy: (i) a smaller and a stronger external magnetic controlling force have been considered, by using a series of two or four permanent magnets of the type S0805 
1 (nominal force $\boldsymbol{F}=19.61 \mathrm{~N}$ each); (ii) the gain of the conditioning circuit $G$, which allows to

2 amplify the output voltage signal, has been varied in the range $G=11.6 \div 25.0$.

Table 5 summarizes the characteristics of the total 83 wave experiments, indicating for each series of tests: the number of tests, the type and number of permanent magnets used to generate the Rosenseweig effect on the ferrofluid, the value of the gain $G$ of the conditioning circuit, the ranges of water depth $h$, wave height $H$ and wave period $T_{\text {wave }}$. Additionally to the experiments performed in the presence of regular surface waves, for each test series a hydrostatic experiment has been carried out. Table 5 reports also the range of the investigated dimensionless wave parameters, namely the Reynolds number of the wave boundary layer $R e_{w}=U_{0} A / v$, the relative roughness $A / k_{N}$, and the maximum Shields parameter $\theta_{\max }=\tau_{\text {omax }} / \rho g(s-1) d_{50}$. In particular, the values of the critical Shields parameter (Soulsby, 1997), which are equal to 0.046 for test series WS001-S003 and to 0.030 for test series WS004-WS006, indicate that in about the 30\% of the tests the threshold of motion of the sediments was exceeded, giving rise to a weakly mobile bed over which moving sediment particles impacts on the ferrofluid sensors.

\section{Results}

\subsection{Steady current results}

The proposed ferrofluid sensor and the inductive readout strategy described in Section 2 have been first tested in steady current conditions over a fixed smooth bottom. These are highly repeatable tests which allow to define the range of application of the system, to investigate the existence of a unique calibration curve and its dependence on the controlling parameters.

Preliminary results proved that the influence of the thermal and electronic instability of the conditioning circuit is negligible, since the maximum uncertainty of repeated measurements is about $0.3 \%$ (Musumeci et al., 2015b). The specific aim of the present experimental campaign has been instead to assess the influence of the intensity of the external controlling magnetic field on the ferrofluid measurements.

Figure 7 shows the relationship between the average output voltage $V_{\text {out }}$ measured by the conditioning circuits and the velocity at the ferrofluid location $U_{F F}$, i.e. the velocity at $1 \mathrm{~mm}$ above the bottom. In particular, the Figure shows the experimental data of repeated tests carried out by using a smaller intensity (nominal force $\boldsymbol{F}=2 \cdot 19.61 \mathrm{~N}$, see Figure 9 a); a medium intensity (nominal force $\boldsymbol{F}=2 \cdot 24.51 \mathrm{~N}$, see Figure 9 b) and a larger intensity (nominal force $\boldsymbol{F}=3 \cdot 19.61$ $\mathrm{N}$, see Figure 9c) of the external controlling magnetic field generated by the permanent magnets (see Table 4). 
When using the weaker magnets (Figure $7 \mathrm{a}$ and $7 \mathrm{~b}$ ), the relationship between the velocity at the ferrofluid $U_{F F}$ and the measured output voltage is consistent within each individual test, as it follows a parabolic shape with small scatter. However, by comparing repeated trials performed in the same hydraulic conditions using the same number and type of magnets, the calibration curves obtained for each tests are different. On the other hand, when a stronger controlling force is exerted on the ferrofluid spike, all the data collapses on the same curve (see Figure 7c). In the presence of weaker retaining magnetic force, the equilibrium condition of the conditioning circuit, and in turn the measured calibration curve, may be affected by small differences in the condition of the experimental set-up (i.e. ambient temperature, salinity of the water, slight change of the electrical input signal, etc.). Moreover, being the ferrofluid spike less stiff (then more deformable) it can assume different possible shapes under the effect of a flow, due to the interaction with the magnetic field lines in the neighbourhood, thus producing different variations of the inductance of the sensing coils. Such spurious effects become less important when the retaining force is larger. The grey area reported in Figure 7 indicates a region within which the sensitivity of measuring system is low. Indeed, when the velocity $U_{F F}$ is small, the action of the external magnetic controlling force prevails on the action of the flow and the ferrofluid spike is only slightly deformed. In principle, the lower limit for the application of the proposed measurement methodology is a function of the external magnetic force and of the volume of the ferrofluid drop. However, as shown in Figure 7, the variability of such a threshold is quite small in the tested conditions and the value $U_{F F}=0.08$ $\mathrm{m} / \mathrm{s}$ is considered reasonable in all cases. It should be mentioned that an upper limit of the flow intensity also exists for the application of the present measurement system. Indeed, if the flow velocity at the ferrofluid tip is too large, the force of the permanent magnets which generates the Rosensweig effect may not be large enough to keep the ferrofluid at the measuring point and the ferrofluid drop can be carried away with the flow. By using the combinations of controlling magnetic forces reported in Table 4, it has been found that when $U_{F F}$ is larger than $0.2 \mathrm{~m} / \mathrm{s}$, the proposed methodology cannot be applied.

Figure 8 reports the relationship between the measured output voltage $V_{\text {out }}$ and the average bottom shear stress $\tau_{x 0}$, for the same test conditions considered in Figure 7. Also in this case a lower limit and an upper limit of the average bottom shear stress are recovered when applying the proposed methodology for measuring bottom shear stresses in the presence of steady currents. In the investigated conditions, such limits are about equal to $0.08 \mathrm{~N} / \mathrm{m}^{2}$ and to $0.2 \mathrm{~N} / \mathrm{m}^{2}$, respectively. In particular, the experimental results demonstrate that the response of the proposed methodology can be assumed to be linear above the lower threshold. Moreover, also in this case, a large scatter is recovered in the presence of weaker intensities of the external controlling magnetic field and the collapse of the experimental data on the same curve is observed when such an intensity is larger. This indicates that the permanent magnetic field 
1 should be strong enough, compared to the flow intensity, to reduce the uncertainties of the

2 measurements and to make the proposed measurement system reliable within its range of application.

\subsection{Regular wave tests}

Regular wave tests in the presence of sandy bed have been performed to analyze (i) the range of application of the system in oscillating flows, particularly in deep, intermediate and shallow water conditions, and (ii) to assess the sensitiveness to changes of the retaining force or of the gain of the circuit. In this case the bed is characterized by a weak mobility (see Table 5). Therefore wave experiments have been aimed also at testing the "survival" of the ferrofluid sensor, notwithstanding the fact that several sand grains impact on the spike. Indeed it is known that hot films probes, for example, are damaged by the action of sediments on the sensor.

In the case of an oscillating flow, values of the bottom shear stress vary between $\tau_{0 \max }$ and $-\tau_{\text {omax }}$ during the wave cycle. Therefore, the testing of the ferrofluid sensor in oscillating conditions must take into account the frequency response of the overall measuring system and the influence of control parameters (e.g. the intensity of the external permanent magnetic field and of the gain of the conditioning circuit).

First, an analysis of the frequency response of the conditioning circuit has been carried out. Figure 9 shows several examples of the raw time-dependent signals of both the surface elevation $\eta$ and of the peak-to-peak output voltage $V_{\text {out }}$ measured by the resistive wave gauge and by the conditioning circuit respectively. The results show that the proposed system is able to provide not only the maxima bottom shear stresses in the presence of oscillatory flow, but also its periodic behaviour. Moreover, it should be observed that the sensitivity of the proposed system increases as wave heights and periods increase.

Figure 10 presents the mean wave periods measured independently by the wave gauge and by the ferrofluid sensor through the conditioning circuit, namely $T_{\text {wave }}$ and $T_{\text {circ }}$, both estimated by averaging the individual wave periods determined by applying a zero-up crossing method. Figure 10 reports also the error bars of $T_{\text {circ }}$, which corresponds to the standard deviation of each test within the test series. The magnet strengths and the circuit gain used in the different test series are the ones reported in Table 5. The experimental results show that the wave period measured by the proposed system is generally consistent with that of the waves, which means that the frequency response of the measuring system is linear in the range of investigated frequencies. It may be observed that the system behaves better in the presence of shallow and intermediate water depth waves, while a larger dispersion of the experimental data is recovered in the presence of waves closer to the deep water limit. In the latter case, the interaction between the wave motion and the bottom, where the ferrofluid is 
1 located, is negligible. The results obtained in deep waters prove that the influence of the

2 changing water level is not significant on the voltage output recovered by the conditioning circuit. On the other hand this increases the probability of errors in the estimate of oscillations of the output signal, due to the residual noise of the conditioning circuit. Moreover, it appears that larger errors in the determination of the wave period occur when a larger gain of the conditioning circuit is adopted, as in the test series WS004 and WS005 ( $G=25.0)$. On the other hand the increase of the strength of the magnetic field play just a minor role. This is probably due to the increased level of noise and the amplification of the disturbances obtained in such a case. Smaller gains guarantee a smaller variability of the measurements (e.g. see test series WS002 and WS003).

The capability of the proposed ferrofluidic sensor to measure bed shear stresses in the presence of regular surface wave is analyzed by comparing the maximum bed shear stress $\tau_{0 \max }$ to the peak-to-peak output voltage of the inductive transducer $V_{p p}$. In particular, Figure 11 shows the relationship between $V_{p p}$ and $\tau_{0 \max }$ for the small and the large value of the external controlling magnetic force, considering a constant gain equal to $G=11.6$. Figure 12 reports analogous data with an increased value of the gain, i.e. $G=25.0$. Finally, keeping constant the intensity of the external magnetic field, Figure 13 compares the behaviour of the conditioning circuit with increasing values of the gain.

The analysis of the experimental data reveals that, also in the presence of the waves, a lower limit of application of the proposed methodology exists, which corresponds to a threshold of the maximum bed shear stress below which the developed ferrofluid sensor is not able to react consistently to the action of the flow. In particular, in the investigated wave conditions, the range of application of the proposed technique is comprised between a lower limit equal to $0.08 \mathrm{~N} / \mathrm{m}^{2}$, which is equal to that recovered in the presence of steady currents, and an upper limit equal to about $0.4 \mathrm{~N} / \mathrm{m}^{2}$, determined by the characteristics of the waves which was possible to generate in the experimental tank.

Coherently with the results shown in the previous Section, also in the presence of waves the relationship between the measured peak-to-peak output voltage $V_{p p}$ and the maximum wave bed shear stress $\tau_{0 \max }$ is linear. In particular, the results in Figure 11 demonstrate that the sensitivity of the developed sensor decreases as the intensity of the external magnetic field increases. Indeed a stronger magnetic field makes the ferrofluid spike stiffer, making it less sensible to the action of the flow. Moreover, in the latter case the repeatability of the measurements is reasonable also in the presence of waves, notwithstanding the less controllable experimental conditions due to the characteristics of the larger experimental apparatus, compared to those of the steady current experiments (see Figure $11 \mathrm{~b}$ ). 
Although a linear trend may generally be recognized, the comparison between Figures 11, 12 and 13 shows that the increase of the gain of the conditioning circuit generates a greater dispersion of the measurements. It follows that for the optimal setup of the system, a gain $G=11.6$ should be used, at least in the investigated experimental conditions.

Finally, besides a large dispersion, the results in Figure 12a and Figure 13b, which correspond to the test series WS004( no. 2 magnets S0805, $G=25.0$ ) and WS006 (no. 4 magnets $\mathrm{S} 0805, G=19.5$ ) respectively, are characterized by an almost constant value of peakto-peak output voltage over the entire range of investigated bed shear stresses. Indeed, in these cases the noise induced by the higher gain of the transducer is larger than the inductive unbalance in the conditioning circuit due to the ferrofluid deformation and displacement, therefore the readout strategy is not able to measure correctly.

5 Discussion

The experimental validation of the new ferrofluid sensor coupled to the developed inductive readout strategy to measure bottom shear stress at the bottom of currents and waves allows to highlight some of the peculiar features of the proposed methodology.

First of all, the working range of the system is determined. Such a range is a function of both the configuration of the measuring system, i.e. of the intensity of the controlling magnetic field, and of the flow characteristics, e.g. steady or oscillatory motion. In particular, in the investigated conditions it is found that it is reasonable to consider the minimum measurable value of bottom shear stress equal to about $0.08 \mathrm{~N} / \mathrm{m}^{2}$ for both currents and regular waves. Indeed, below such a threshold, the magnetic field which generates the Rosensweig effect on the ferrofluid drop is stronger than the action of the flow, thus the deformation of the ferrofluid drop induced by the latter is too small to be sensed by the conditioning circuit and transduced into a coherent output voltage. On the other hand, the upper limit of the working range of the measurement system is different in the case of currents and waves. In the first case, the upper limit is equal to $0.2 \mathrm{~N} / \mathrm{m}^{2}$, while it is doubled to a value of about $0.4 \mathrm{~N} / \mathrm{m}^{2}$ in the second case. It should be observed that the latter value is determined as a mere consequence of the characteristics of the highest/longest waves generated experimentally within the wave tank. Indeed, in this case the ferrofluid drop is always kept at the measuring point and it is not washed away with the flow, therefore the above threshold could be, in principle, even larger.

Such a difference should be indeed expected, as a consequence of the oscillatory nature of the wave motion. In fact, in the steady flow case an average bed shear stress is measured, but due to the turbulence the instantaneous values of the bottom shear stress could 
1 be much higher than the mentioned average threshold. Instead, under the oscillating wave motion the maximum flow action on the ferrofluid spike, which corresponds to the estimated value of $\tau_{0 \max }$, is exerted only for few instants during the wave cycle, namely during the crest and trough phase. It follows that the ferrofluid sensor can be controlled at the measuring station even in the presence of relatively more intense flow conditions, before being carried away.

Another important element for the setup of the proposed measuring system is related to the amplification of the output voltage signal, which can be obtained by changing the value of the gain. Indeed, in the presence of steady flow larger gains can be used (here $G=277$ is considered in all the steady current tests) without introducing significant noise in the measurements. The operations in the presence of waves require a more careful setting of the system and the use of much smaller values to avoid undesired dispersion of the experimental data. In the present experimental wave conditions, the optimal value is equal to $G=11.6$.

In the above ranges of application and considering an optimized gain of the conditioning circuit, the calibration curve of the ferrofluid sensor turns out to be significantly linear in both steady current and wave conditions.

Finally, it is found that the sensitivity of the measuring system is controlled by the intensity of the permanent magnetic field used to generate the ferrofluid spike through the Rosensweig effect. However the system behaves differently, depending on the flow type.

Figure 14 reports the calibration curves of the system obtained in terms of the measured average output voltage $V_{\text {out }}$ and estimated average current bottom shear stress $\tau_{x 0}$ in the flow direction, for different values of the permanent magnetic field. The $50 \%$ confidence interval is also plotted. Similar calibration curves of the peak-to-peak output voltage $V_{p p}$ and of the maximum wave bed shear stress $\tau_{0 \max }$ and confidence intervals are presented in Figure 15 for the wave case. Moreover, for both flow types, Table 6 summarizes the calibration parameters and the errors, in terms of the slope $S$ of the linear calibration curve and of the minimum and maximum standard deviation $s d$ of the predictions with respect to such a calibration curve. It is worth pointing out that a smaller value of $S$ corresponds to a larger sensitivity of the measuring system.

In steady current conditions, only a minor reduction of sensitivity is registered as the intensity of the magnetic field increases. However larger difference appears when considering the errors of the measurements. Indeed, the values of the standard deviation decrease as the intensity of the magnetic field increases. In particular, considering the present experimental campaign, when the magnetic field is weaker, some errors larger than one standard deviation are recovered in the dataset, while the use of stronger magnetic field guarantees errors equal to about $0.1 s d$. It turns out that if the intensity of the retaining force is properly selected 
1 compared to the flow conditions, the width of the confidence interval may be significantly reduced, up to a factor of three in the case being (e.g. compare Figures 14a and 14b to Figure $14 c)$.

In the presence of regular waves, the sensitivity of the proposed measuring system changes significantly compared to the steady flow case, with values of $S$ equal to 2 or 3 times those observed in the latter condition. Again the measurement sensitivity was increased by decreasing the strength of the magnetic field. However, as opposite to the current case, the measurement errors are smaller if the magnetic field is smaller. Indeed, under the oscillating motion the ferrofluid spike must move continuously back and forth under the action of a varying flow, between $\tau_{0 \max }$ and $-\tau_{0 \max }$ in half of a wave period. If the controlling magnetic field is too strong it may contrasts such a smooth movement, by producing larger standard deviation values and a larger dispersion of data (see Figure 15b).

Finally some considerations should be made about the use of the system in the presence of sediments. As mentioned in Section 3.2, during the tests performed within the wave tank, the measuring apparatus is buried within the sand. In particular, two types of sediments are used, a medium-coarse quartz sand $\left(d_{50}=0.56 \mathrm{~mm}\right)$ and a medium fine $\left(d_{50}=0.24\right.$ $\mathrm{mm}$ ) quartz sand. According to Soulsby (1997), the value of the critical bed stress for the incipient motion of the sediment is about $0.27 \mathrm{~N} / \mathrm{m}^{2}$ and $0.17 \mathrm{~N} / \mathrm{m}^{2}$, since the critical Shields parameter $\theta_{c}$ is about 0.046 and 0.030 respectively. It follows that, at least in the case of the finer sand, there should be a weak bed load during part of the wave cycle, with sand particles impacting on the ferrofluid drop. Since the experimental data are not significantly affected by such impacts (see, for example, Figure 11), the experimental campaign carried out in the presence of sediments confirms that the proposed measurement methodology is robust enough to be used in the presence of a weak bedload transport without costly damages to the sensor.

\section{Conclusions}

In the present work the development of a system for measuring bottom shear stresses generated by waves and currents based on the use of ferrofluids is presented.

The Rosensweig effect is used to generate a single peak of ferrofluid right at the bottom, whose dimensions are $\mathrm{O}(1 \mathrm{~mm})$ and which acts as a sensor of the bottom shear stresses, being deformed and misplaced by the action of the flow.

An inductive readout strategy is used to sense the movement of the center of mass of the ferrofluid spike as a modification of the inductance produced by two planar coils. Such coils are photoligraphically engraved onto a printed circuit board, located at the bottom and 
controlled by a conditioning circuit, whose direct current output voltage can be used as a measure of the bottom shear stress.

The sensing element is relatively small $\mathrm{O}(1 \mathrm{~mm})$ and can provide measurements very close to the wall. Thanks to the dimensions of the ferrofluidic sensing element and to the robustness of the electro-magnetic transducer, the proposed technique is complementary to state-of-the-art instruments, such as hot-film and MEMS, since it overcomes some of their limits, mainly related to the costs and to the fragility of the sensors in the presence of sediments moving at the bottom.

The proposed measurements methodology has been experimentally tested to obtain measurements of the bottom shear stress in the presence of both steady currents and surface regular waves. In the latter case, the measuring system is located over a sandy bottom characterized in dynamic conditions by a weak bedload transport.

In all the investigated flow conditions the behaviour of the proposed ferrofluid sensor to measure bottom shear stresses is reasonably linear, while the sensitivity of the measuring system and the related errors are function of the external magnetic field used to generate the Rosensweig effect and to control the ferrofluid spike at the measuring station. Indeed, by increasing the strength of the magnetic field also the stiffness of the ferrofluid spikes increases. This may lead to relatively large scatter of the data, if the strength of the magnetic field is not large enough.

Moreover, also the range of measured velocities is a function of such a parameter. Indeed, the smallest measurable value of bottom shear stress is about $0.08 \mathrm{~N} / \mathrm{m}^{2}$ in all analyzed conditions, while the largest measurable value is different in the presence of currents or waves. In the first case, a maximum value of about $0.2 \mathrm{~N} / \mathrm{m}^{2}$ can be measured, before the ferrofluid drop is dragged away. In the presence of the oscillating motion, larger values of measured velocity and bottom shear stresses can be reached. This is due to the fact that in the present tests only laminar/transitional oscillatory flow conditions have been generated. Therefore the maximum value of such variable is achieved only for few instants during the wave cycle.

A limitation of the technique is related to the fact that only information on the mean flow can be obtained, due to the relatively large frequency response of the ferrofluid sensor $\mathrm{O}(5 \mathrm{~Hz})$ compared to that of turbulent velocities.

Furthermore, in the present work, the calibration of the system relies on the measured or theoretical knowledge of the velocity profile. A direct calibration, with no assumptions on the flow structure is still desirable for a practical application of the proposed methodology. Therefore, a future work will be to compare measurements obtained using the ferrofluid sensor with those obtained by state of the art instruments, such as shear plates or hot-films. Moreover future developments of the proposed technique will be aimed at increasing the 
1 measuring range, which is mainly related to the intensity of the permanent magnetic field. A

2 possible solution could be to use self-adjusting magnetic strength, by implementing a

3 measuring closed-loop feedback response. To these aims, the testing of the proposed

4 methodology in a large scale setup is considered necessary. By taking advantage of the non-

5 invasiveness of the measuring system, other possible developments include the possibility to

6 obtain measurements in the presence of biologically active beds, such as vegetated bottoms.

\section{$7 \quad$ Acknowledgements}

8 The authors wish to thanks the research group of the Joint Research Activity- WISE of the

9 HYDRALAB IV project for the many helpful and stimulating discussion on the development

10 of the proposed measured strategy.

\section{Funding}

12 This work has been partly funded by the European Commission projects Hydralab IV

13 (contract no. 261520) and Hydralab Plus (proposal no. 654110).

\section{Notation}

$15 A=$ wave oscillations amplitude at the bottom

$16 \quad d_{50}=$ median diameter $(\mathrm{mm})$

$17 \boldsymbol{F}=$ nominal magnetic attraction force $(\mathrm{N})$

$18 f_{w}=$ wave friction factor $(-)$

$19 G=$ gain of the conditioning circuit (-)

$20 \quad H=$ surface wave height $(\mathrm{mm})$

$21 h=$ water depth $(\mathrm{m})$

$22 \quad k_{N}=$ Nikuradse roughness $(\mathrm{m})$

$23 L=$ inductance $(\mathrm{H})$

$24 \quad L_{l}=$ inductance of one of the planar coils $(\mathrm{H})$

$25 L_{2}=$ inductance of one of the planar coils $(\mathrm{H})$

$26 \quad L_{\text {wave }}=$ wavelength of the surface wave $(\mathrm{m})$

$27 \quad R e=$ Reynolds number $(-)$

$28 R e_{w}=$ Reynolds number of the wave boundary layer (-)

$29 S=$ slope of the linear calibration curve (-)

$30 s d=$ standard deviation of the prediction with respect to the linear calibration curve $\left(\mathrm{N} \cdot \mathrm{m}^{-2}\right)$

$31 \quad T_{\text {circ }}=$ period measured by the conditioning circuit (s)

$32 T_{\text {wave }}=$ surface wave period (s)

$33 t=$ time $(\mathrm{s})$ 
$1 \quad U=$ mean velocity in the flow direction $\left(\mathrm{m} \cdot \mathrm{s}^{-1}\right)$

$2 \bar{U}=$ depth-averaged mean velocity in the flow direction $\left(\mathrm{m} \cdot \mathrm{s}^{-1}\right)$

$3 U_{0}=$ amplitude of the wave potential velocity at the bottom in the wave direction $\left(\mathrm{m} \cdot \mathrm{s}^{-1}\right)$

$4 \quad U_{F F}=$ mean velocity at the ferrofluid location $\left(\mathrm{m} \cdot \mathrm{s}^{-1}\right)$

$5 \quad u^{*}=$ friction velocity $\left(\mathrm{m} \cdot \mathrm{s}^{-1}\right)$

$6 V_{\text {out }}=$ voltage output of the conditioning $\operatorname{circuit}(\mathrm{V})$

$7 \quad V_{p p}=$ peak to peak output voltage of the conditioning circuit $(\mathrm{V})$

$8 x=$ horizontal coordinate, positive in the flow direction $(\mathrm{m})$

$9 y=$ dimensional distance from the bottom $(\mathrm{m})$

$10 y^{+}=$dimensionless distance from the bottom (-)

$11 \delta=$ thickness of the wave boundary layer $(\mathrm{mm})$

$12 \eta=$ surface elevation $(\mathrm{cm})$

$13 \kappa=$ constant of von Karman (-)

$14 \mu=$ magnetic permeability $\left(\mathrm{H} \cdot \mathrm{m}^{-1}\right)$

$15 v=$ kinematic viscosity $\left(\mathrm{m}^{2} \cdot \mathrm{s}^{-1}\right)$

$16 \theta=$ Shields parameter (-)

$17 \theta_{c}=$ critical Shields parameter (-)

$18 \rho=$ density $\left(\mathrm{kg} \cdot \mathrm{m}^{3}\right)$

$19 \sigma=$ wave angular frequency $\left(\mathrm{m}^{-1}\right)$

$20 \tau_{c}=$ critical bed shear stress $\left(\mathrm{N} \cdot \mathrm{m}^{-2}\right)$

$21 \tau_{x 0}=$ bottom shear stress in the flow direction $\left(\mathrm{N} \cdot \mathrm{m}^{-2}\right)$

$22 \tau_{\text {omax }}=$ maximum bottom shear stress under oscillatory flow $\left(\mathrm{N} \cdot \mathrm{m}^{-2}\right)$

\section{References}

Achenbach, E. (1968). Distribution of local pressure and skin friction around a circular cylinder in cross-flow up to $\mathrm{Re}=5 \times 10^{5}$. J. Fluid Mech., 34(4), 625-639.

Ackerman, J. D., \& Hoover, T. M. (2001). Measurement of local bed shear stress in streams using a Preston-static tube. Limnology and Oceanography, 46(8), 2080-2087. measurement of velocity profiles and shear stresses in boundary layers. IEEE Sensors 2009 Conference. 
Andò, B., Baglio, S., \& Beninato, A. (2012). A low-cost inertial sensor based on shaped magnetic fluids. IEEE Transactions on Instrumentation and Measurement, 61(5), 12311236.

Bagherimiyab, F., \& Lemmin, U. (2013). Shear velocity estimates in rough-bed open-channel flow. Earth Surface Processes and Landforms, 38(14), 1714-1724.

Barnes, M. P., \& Baldock, T. E. (2007). Direct bed shear stress measurements in laboratory swash. J. Coastal Res., 50, 641-645.

Cox, D. T., Kobayashi, N., \& Okayasu, A. (1996). Bottom shear stress in the surf zone. $J$. Geoph. Res. 101(C6), 14337-14348.

Cowley, M. D., \& Rosensweig, R. E. (1967). The interfacial stability of a ferromagnetic fluid. J. Fluid Mech., 30(4), 671-688.

Foti, E., Faraci, C., Foti, R., \& Bonanno, G. (2010). On the use of bioluminescence for estimating shear stresses over a rippled seabed. Meccanica 45(6), 881-895.

Gust, G. (1988). Skin friction probe for field applications. J. Geoph. Res. 93(C11), 1412114132.

Head, M. R., \& Rechenberg, I. (1962). The Preston tube as a mean of measuring skin friction. J. Fluid Mech. 14(01), 1-17.

Löfdhal, L., \& Gad-el-Hak, M. (1999). MEMS-based pressure and shear-stress sensor for turbulent flows. Meas. Sci. Technol. 10, 665-686.

Musumeci, R. E., Cavallaro, L., Foti, E., Scandura, P., \& Blondeaux, P. (2006). Waves plus currents crossing at a right angle: Experimental investigation. J. Geoph. Res. 111, C07019 1- C07019 19.

Musumeci, R. E., Marletta, V., Andò, B., Baglio, S., \& Foti, E. (2015a). Measurement of wave near-bed velocity and bottom shear stress by ferrofluids. IEEE Transaction on Instrumentation and Measurement, 64(5), 1232-1239.

Musumeci, R. E., Marletta, V., Andò, B., Baglio, S., \& Foti, E. (2015b). Ferrofluid measurements of bottom velocities and shear stresses. J. Hydrodynamics, 27(1), 150158.

Myrhaug, D. (1989). A rational approach to wave friction coefficients for rough, smooth and transitional turbulent flow. Coastal Eng., 13, 11-21.

Naughton, J. W., \& Sheplak, M. (2002). Modern developments in shear-stress measurement. Progr. Aerosp. Sci, 38, 515-570.

Nimmo Smith, W. A. M., Atsavapranee, P., Katz, J., \& Osborne, T. R. (2002). PIV measurements in the bottom boundary layer of the coastal ocean. Exps. in Fluids 33, 962-971.

Odenbach, S. (2002). Ferrofluids: magnetically controllable fluids and their applications. Springer-Verlag. 
1 Odenbach, S. (2004). Recent progress in magnetic fluid research. J. Phys.: Condensend Matter, 16, R1135-R1150.

Okamoto, T., Yagita, M. (1977). Flow past cone placed on flat plate. Bulletin of the JSME, $20,141,329-336$.

Rankin, K. L., \& Hires, R. I. (2000). Laboratory measurement of bottom shear stress on a movable bed. J. Geoph. Res.105(C7), 17011-17019.

Rezazadeh, G., Loftiani, A., \& Khalilarya, S. (2009). On the modelling of a MEMS-based capacitive wall shear stress sensor. Measurement 42, 202-207.

Riedel, H. P., Kamphuis, J. W., \& Brebner, A. (1974). Measurement of bed shear stress under waves. In Proc. 14 ${ }^{\text {th }}$ Int. Conf. on Coastal Eng., Copenaghen, Denmark 587-603.

Roszkowski, A., Bogdan, M., Skczynski, W., Marek, B. (2008). Testing viscosity of MR fluid in magnetic field. Measurement Science Review, 8, 3 (3), 58-60.

Soulsby, R. (1997). Dynamics of marine sands. Thomas Telford Publications, London, UK.

Sumer, B. M., Arnskov, M. M., Christiansen, N., \& Jorgensen, F. E. (1993). Two-component hot-film probe for measurements of wall shear stress. Exp. in Fluids 15, 380-384.

Sumer, B. M., Fredsoe, J. (1997). Hydrodynamics around cylindrical structures. World Scientific.

Wallace, J. M., \& Vukoslavčević, P.V. (2010). Measurement of the velocity gradient tensor in turbulent flows. Ann. Rev. Fluid Mech., 42, 157-181.

Winter, K. G. (1977). An outline of the techniques available for the measurement of skin friction in turbulent boundary layers. Progr. Aerosp. Sci.18, 1-57.

You, Z-J., Yin, B.S., Huo, G. (2009). Direct measurements of wave induced bottom shear stress under irregular waves. In Advances in Water Resources and Hydraulic Engineering, Springer, 1213-1218. 
Tables

2 
Table 1 Summary of the main characteristics of state-of-the-art instruments used to measure bottom shear stresses, compared to the proposed ferrofluid sensor.

\begin{tabular}{|c|c|c|c|}
\hline Instrument type & Principle of Operation & Advantages & Disadvantages \\
\hline Preston and Stanton tubes & Pitot tube & Robustness & $\begin{array}{l}\text { Tube dimensions must be smaller than } \\
\text { wall layer } \\
\text { Difference between total and static pressure } \\
\text { must be large }\end{array}$ \\
\hline $\begin{array}{l}\text { Velocimetry (ADVs, LDVs } \\
\text { and PIVs) }\end{array}$ & $\begin{array}{l}\text { Bottom shear stresses derived } \\
\text { from theoretical approaches } \\
\text { (e.g. log-fit, momentum integral } \\
\text { method, etc.) }\end{array}$ & Reliability & $\begin{array}{l}\text { ADVs and PIVs cannot measure close to } \\
\text { the bottom } \\
\text { Optical instruments difficult to be used } \\
\text { in large flumes } \\
\text { Difficulties in the presence of sediments } \\
\text { High cost }\end{array}$ \\
\hline Shear plates & $\begin{array}{l}\text { Force measurement over } \\
\text { an area } \mathrm{O}\left(0.025 \div 0.543 \mathrm{~m}^{2}\right)\end{array}$ & $\begin{array}{l}\text { Direct measure } \\
\text { Wet-dry conditions can be } \\
\text { handled }\end{array}$ & $\begin{array}{l}\text { Errors due to misalignment, } \\
\text { vibration, thermal expansion, etc. } \\
\text { Trade-off between sensor spatial resolution } \\
\text { and ability to measure small forces }\end{array}$ \\
\hline $\begin{array}{l}\text { Micro-Electro-Mechanical } \\
\text { System (MEMS) }\end{array}$ & $\begin{array}{l}\text { Force measurement over a small } \\
\text { area. }\end{array}$ & $\begin{array}{l}\text { Resolve shear stress of } \\
\mathrm{O}(1 \mathrm{mPa})\end{array}$ & $\begin{array}{l}\text { Errors due to misalignment, pressure- } \\
\text { gradients, cross- acceleration; } \\
\text { Fragility of the sensor }\end{array}$ \\
\hline $\begin{array}{l}\text { Thermal sensors (hot film } \\
\text { probes) }\end{array}$ & $\begin{array}{l}\text { Constant Temperature Anemometers } \\
\text { Area of the sensing elements } \\
\mathrm{O}\left(1 \div 50 \mathrm{~mm}^{2}\right)\end{array}$ & $\begin{array}{l}\text { Ability to measure turbulent } \\
\text { fluctuations }\end{array}$ & $\begin{array}{l}\text { High fragility of the sensor } \\
\text { Difficulties in obtaining unique calibration } \\
\text { relationships } \\
\text { Sensitivity reduction in the dynamic response } \\
\text { Errors due to temperature drift } \\
\text { High cost }\end{array}$ \\
\hline Ferrofluid sensors & $\begin{array}{l}\text { Rosensweig effect } \\
\text { Sensing element volume } \mathrm{O}\left(10 \mathrm{~mm}^{3}\right)\end{array}$ & $\begin{array}{l}\text { Robustness of the sensor } \\
\text { Low cost }\end{array}$ & Cannot measure turbulent fluctuations \\
\hline
\end{tabular}


3 Table 2 Characteristic of the permanent magnets used to generate the Rosensweig effect.

4

5 Type $\boldsymbol{F}$

6

7

$8 \quad \mathrm{~S} 0302 \quad 2.45$

$\begin{array}{lll}9 & \mathrm{~S} 0502 & 6.37\end{array}$

$\begin{array}{lll}10 & \mathrm{~S} 0407 & 6.57\end{array}$

$\begin{array}{lll}11 & \mathrm{~S} 0505 & 8.82\end{array}$

$\begin{array}{lll}12 & \mathrm{~S} 0410 & 10.79\end{array}$

$13 \quad \mathrm{~S} 0606 \quad 12.75$

$14 \quad \mathrm{~S} 0805 \quad 19.61$

$15 \quad \mathrm{~S} 0808 \quad 24.51$

16

17

18 


\begin{tabular}{|c|c|c|c|c|c|}
\hline Flow & $h$ & $\bar{U}$ & $U_{F F}$ & $\tau_{x 0}$ & $\operatorname{Re}$ \\
\hline condition & $(m)$ & $(\mathrm{m} / \mathrm{s})$ & $(\mathrm{m} / \mathrm{s})$ & $\left(N / m^{2}\right)$ & \\
\hline $\mathrm{C} 0$ & 0.110 & - & - & - & - \\
\hline $\mathrm{C} 1$ & 0.110 & 0.03 & 0.01 & 0.004 & 3207 \\
\hline $\mathrm{C} 2$ & 0.110 & 0.05 & 0.02 & 0.010 & 5401 \\
\hline $\mathrm{C} 3$ & 0.110 & 0.08 & 0.04 & 0.017 & 8528 \\
\hline $\mathrm{C} 4$ & 0.110 & 0.10 & 0.06 & 0.038 & 11462 \\
\hline $\mathrm{C} 5$ & 0.110 & 0.12 & 0.07 & 0.051 & 13191 \\
\hline C6 & 0.110 & 0.14 & 0.08 & 0.066 & 15029 \\
\hline $\mathrm{C} 7$ & 0.110 & 0.15 & 0.10 & 0.083 & 17008 \\
\hline $\mathrm{C} 8$ & 0.110 & 0.18 & 0.11 & 0.096 & 19516 \\
\hline C9 & 0.105 & 0.19 & 0.13 & 0.123 & 21003 \\
\hline $\mathrm{C} 10$ & 0.105 & 0.22 & 0.15 & 0.146 & 23363 \\
\hline $\mathrm{C} 11$ & 0.105 & 0.24 & 0.16 & 0.172 & 25863 \\
\hline $\mathrm{C} 12$ & 0.105 & 0.26 & 0.19 & 0.200 & 27489 \\
\hline
\end{tabular}

20

21 


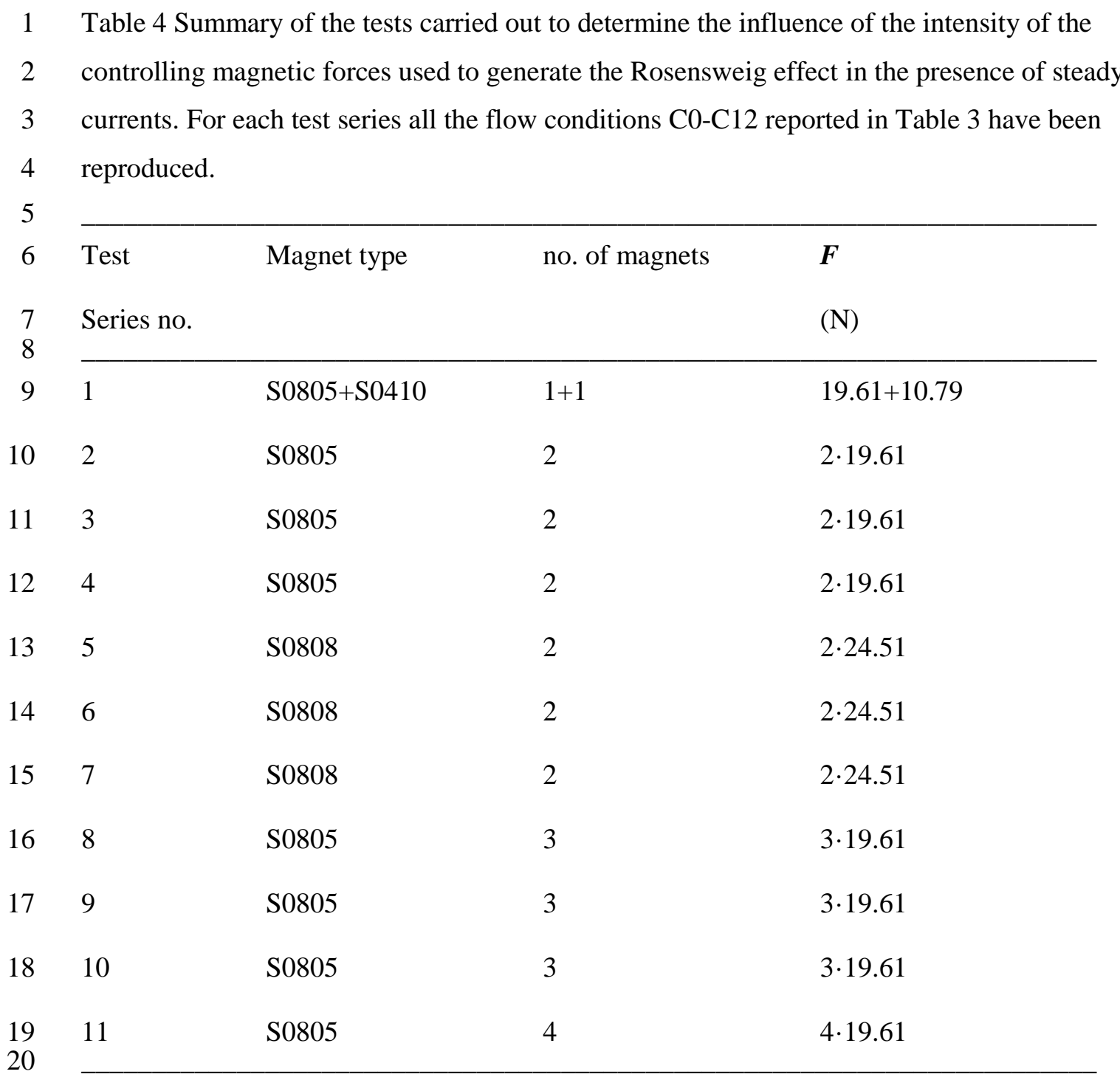

21 
1

2

3

4

5

7

8

8
9

9
10

11

\section{2}

13

14

15

16

17

18

Table 5 Summary of the characteristics and of the dimensionless parameters of the experiments carried out in the presence of waves. The number inside the bracket close to the name of the test series indicates the number of tests which have been carried out. The experiments WS001-S003 have been carried out in the presence of sands with $d_{50}=0.56 \mathrm{~mm}$, the experiments WS004-WS006 have been performed by using sand with $d_{50}=0.24 \mathrm{~mm}$. The critical Shields parameter is equal to 0.046 for test series WS001-S003 and to 0.030 for test series WS004-WS006.

\begin{tabular}{|c|c|c|c|c|c|c|c|c|c|}
\hline \multirow[t]{2}{*}{ Test series } & \multicolumn{2}{|c|}{ Magnets } & \multirow{2}{*}{$\begin{array}{l}\text { Gain } \\
G(-)\end{array}$} & \multicolumn{3}{|c|}{ Wave characteristic ranges } & \multicolumn{3}{|c|}{ Dimensioneless parameters } \\
\hline & Type & no. & & $h(m)$ & $H(\mathrm{~cm})$ & $T_{\text {wave }}(s)$ & $\operatorname{Re}_{w}$ & $A / k_{N}$ & $\theta_{\max }$ \\
\hline WS001(7) & S0805 & 4 & 11.6 & 0.25 & $0.44 \div 4.54$ & $0.37 \div 1.00$ & $0 \div 1401$ & $0.002 \div 10.643$ & $0.000 \div 0.026$ \\
\hline WS002(10) & S0805 & 4 & 11.6 & 0.25 & $0.13 \div 5.36$ & $0.20 \div 1.02$ & $0 \div 2043$ & $0.000 \div 12.857$ & $0.000 \div 0.032$ \\
\hline WS003(11) & S0805 & 2 & 11.6 & 0.25 & $0.06 \div 6.02$ & $0.10 \div 1.03$ & $0 \div 2887$ & $0.000 \div 15.286$ & $0.000 \div 0.038$ \\
\hline WS004(13) & S0805 & 2 & 25.0 & 0.30 & $2.33 \div 5.42$ & $0.51 \div 1.34$ & $2 \div 1819$ & $0.833 \div 28.333$ & $0.002 \div 0.069$ \\
\hline WS005(21) & S0805 & 4 & 25.0 & $0.20 \div 0.30$ & $1.70 \div 3.88$ & $0.67 \div 2.01$ & $69 \div 2935$ & $5.500 \div 36.33$ & $0.013 \div 0.088$ \\
\hline WS006(21) & S0805 & 4 & 19.5 & 0.30 & $1.88 \div 6.66$ & $0.55 \div 1.99$ & $4 \div 2278$ & $1.333 \div 31.667$ & $0.003 \div 0.078$ \\
\hline
\end{tabular}


2 Table 6 Summary of the calibration parameters and of errors.

3$$
4
$$$$
5
$$

6

7$$
8
$$

13

14

15

16

17 

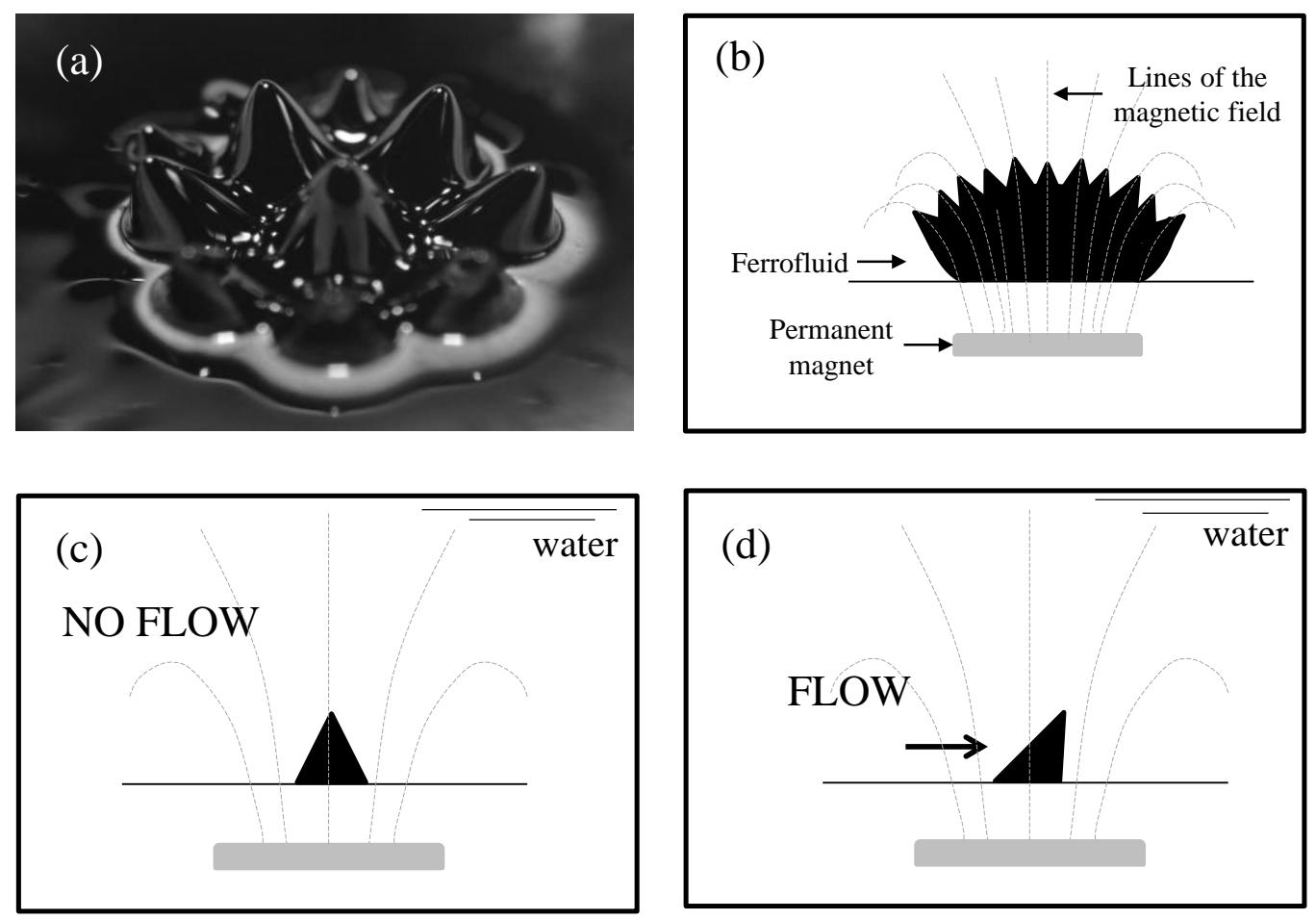

2

3 Figure 1 (a) Example of the Rosensweig effect induced by the action of a magnetic field on a 4 relatively large amount of ferrofluid $\mathrm{O}(1 \mathrm{cl})$; (b) Scheme of such an effect which shows how 5 each single hexagonal conic pattern is aligned along the magnetic field lines; (c) Hydrostatic 6 equilibrium of a single ferrofluid spike; (d) Dynamic equilibrium of a single ferrofluid spike 7 under the action of the flow. 


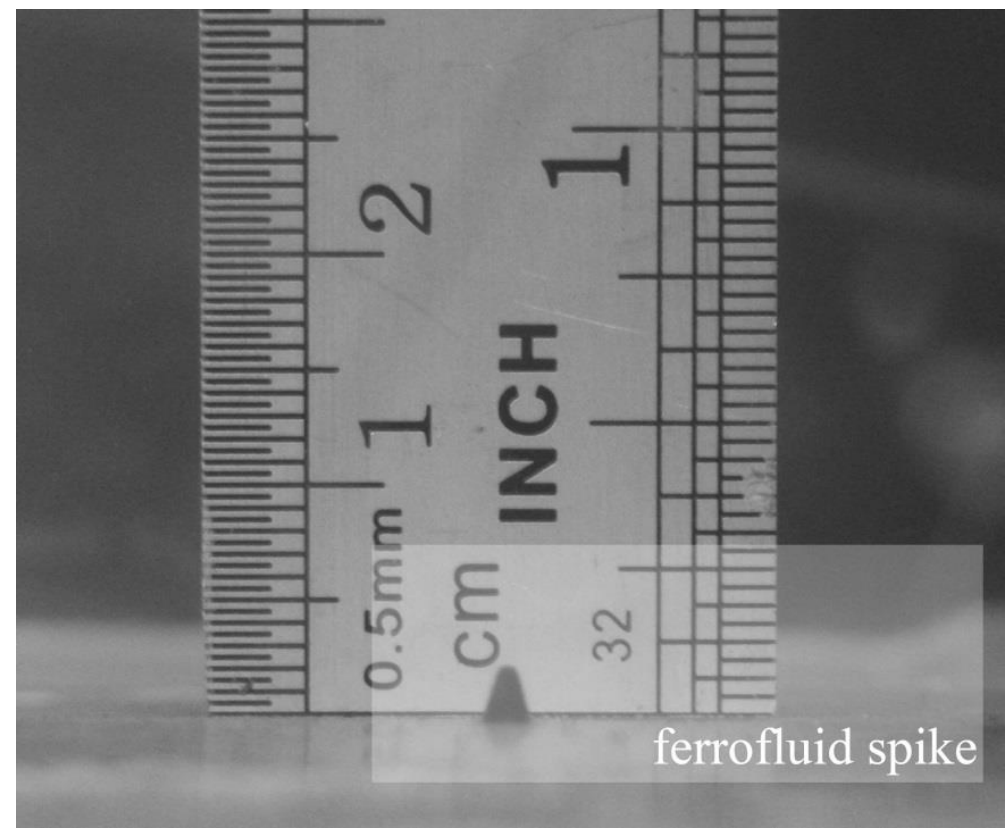

2 Figure 2 Ferrofluid spike generated by the Rosensweig effect at the bottom of the

3 experimental flume, $\mathrm{O}(0.01 \mathrm{ml})$, in the presence of water. The smallest distance between

4 ruler's ticks on the right is $0.5 \mathrm{~mm}$.

5

6 
(a)

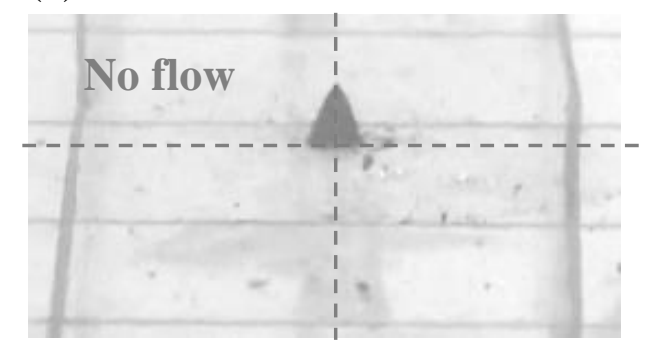

(b)

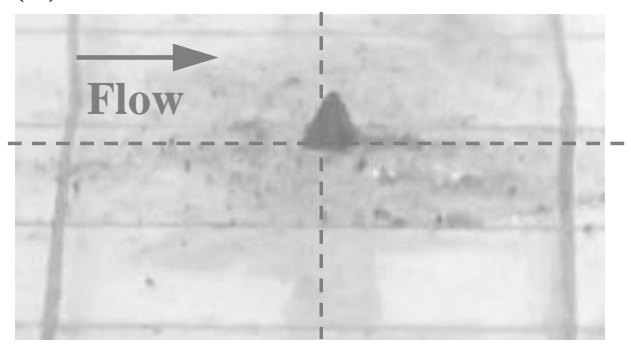

(c)

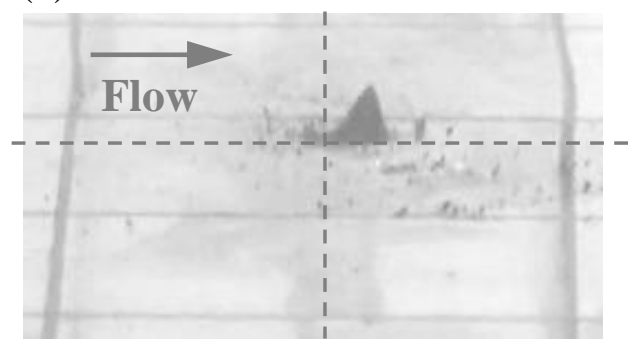

1

2 Figure 3 Ferrofluid spike in: (a) hydrostatic conditions; (b) dynamic conditions (mean

3 velocity $U=0.18 \mathrm{~m} / \mathrm{s}$; velocity at the ferrofluid tip $U_{F F}=0.11 \mathrm{~m} / \mathrm{s}$, bottom shear stress in the

4 flow direction $\tau_{x 0}=0.096 \mathrm{~N} / \mathrm{m}^{2}$ ); (c) dynamic conditions (mean velocity $U=0.26 \mathrm{~m} / \mathrm{s}$; velocity

5 at the ferrofluid tip $U_{F F}=0.19 \mathrm{~m} / \mathrm{s}$, bottom shear stress in the flow direction $\tau_{x 0}=0.200 \mathrm{~N} / \mathrm{m}^{2}$ ). 
(a)

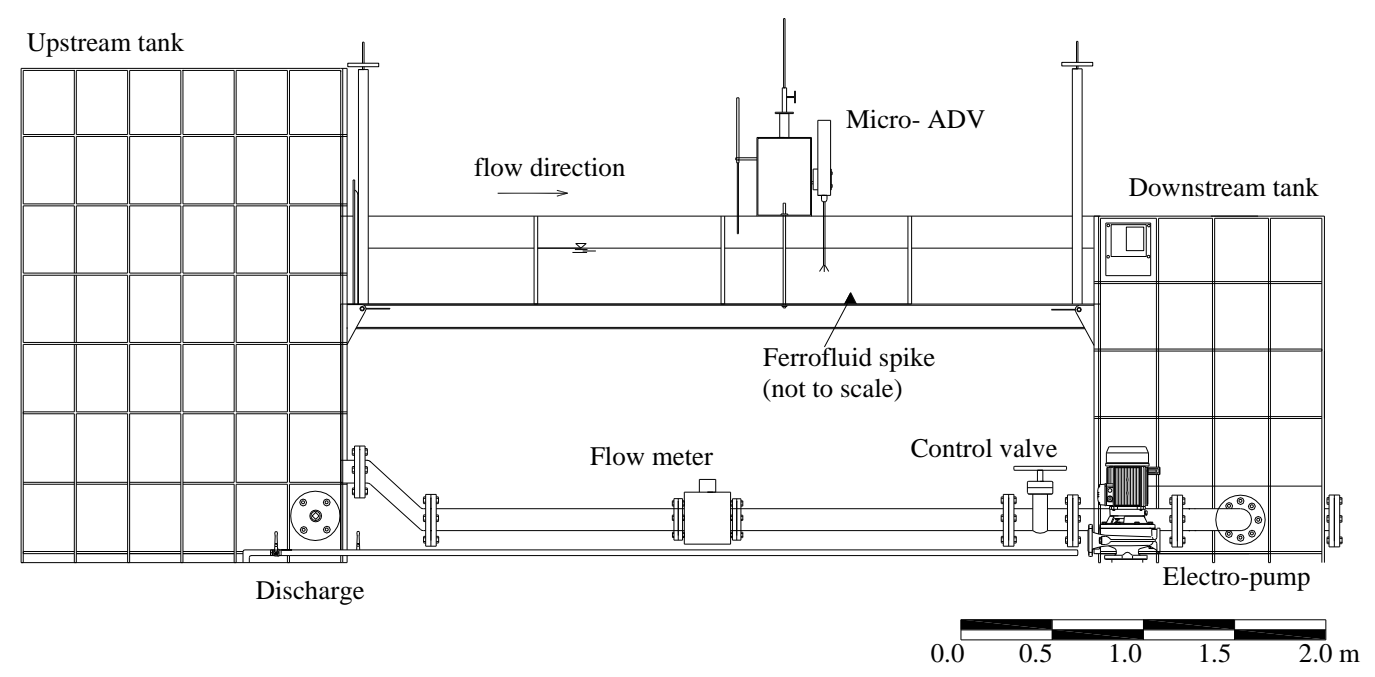

(b)

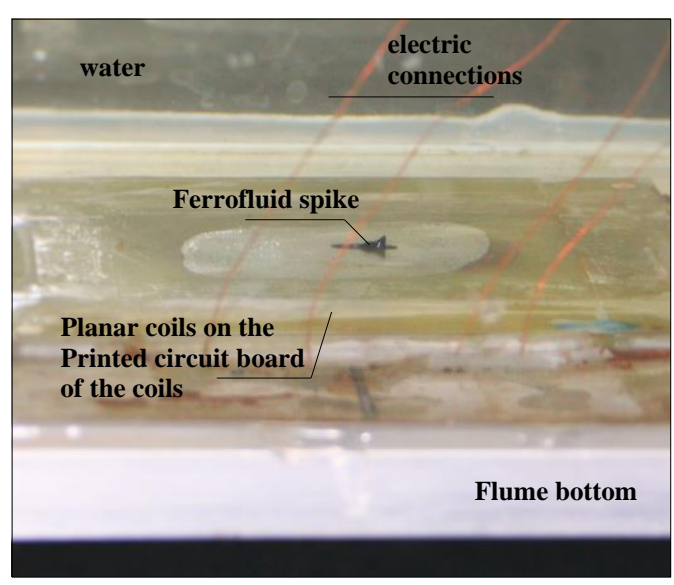

3

4 Figure 4 Experimental setup used for the steady current tests: (a) section of the small scale 5 flume; (b) setup of the ferrofluid sensor with the printed circuit board glued onto the fixed 6 PMMA bottom of the flume (the flow goes from left to right).

7 

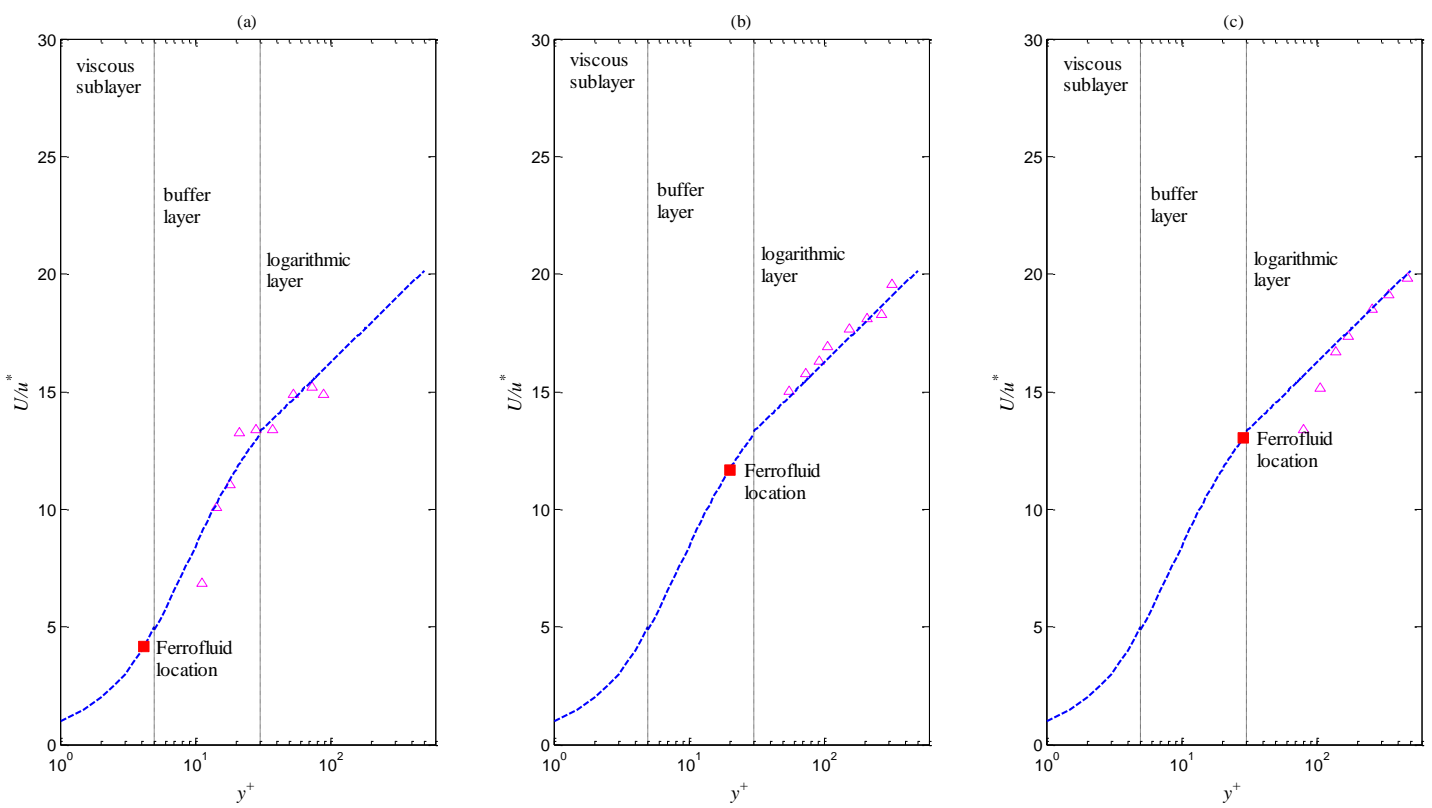

2 Figure 5 Dimensionless theoretical velocity profile within the viscous sublayer, the buffer

3 layer and the logarithmic layer (dashed blue line) and data measured by the micro-ADV. The

4 value of $u^{*}$ is determined by best fitting the measurements on the theoretical profile.

5 Depending on the flow condition, the ferrofluid may located within the: (a) viscous sublayer 6 (flow condition: C1); (b) buffer layer (flow condition:C8); (c) close to the logarithmic layer 7 (flow condition: C12). 
(a)

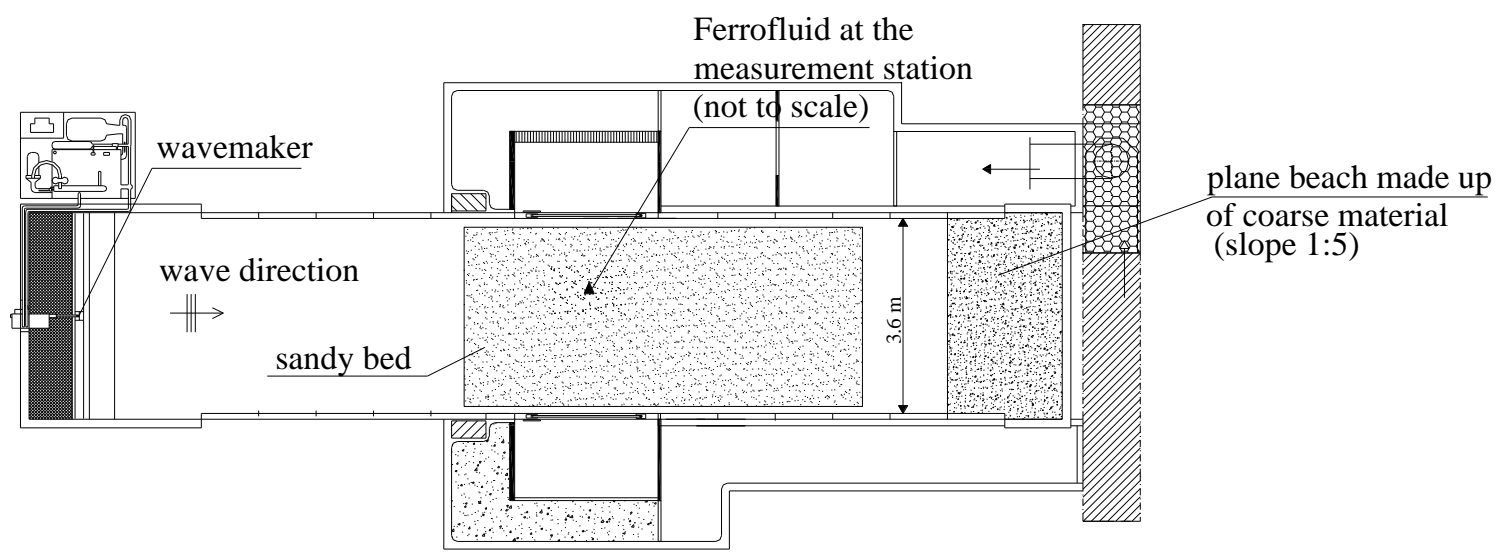

(b)
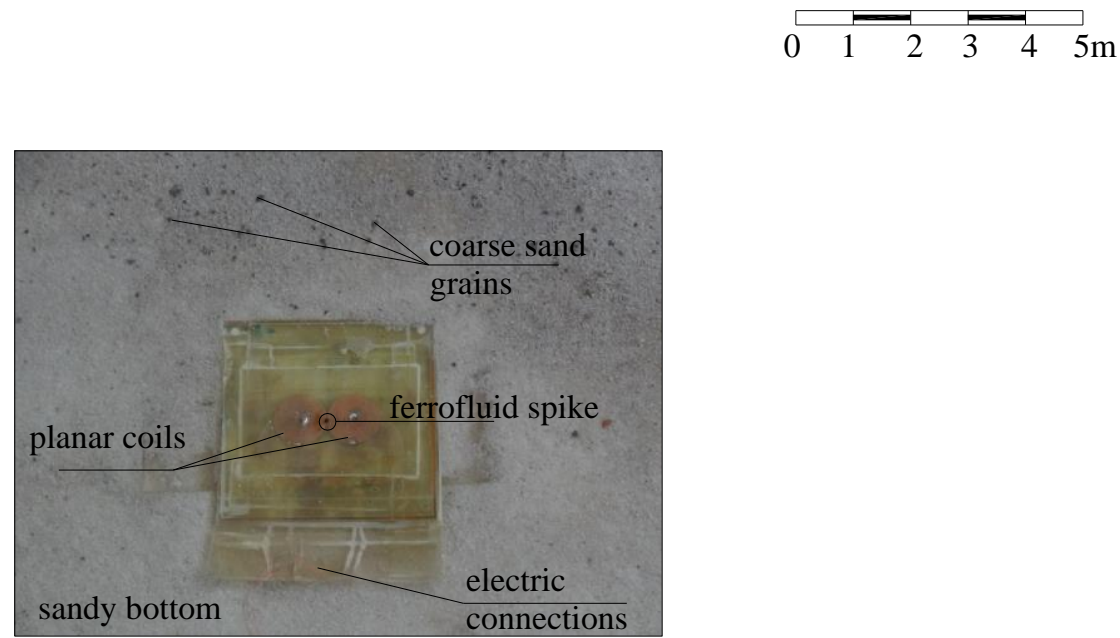

1

2 Figure 6 Experimental setup used for the monochromatic surface wave tests: (a) planar view

3 of the wave tank and location of the measuring point and (b) setup of the ferrofluid sensor

$4 \quad$ with the printed circuit board buried within the sandy bottom $\left(d_{50}=0.56 \mathrm{~mm}\right)$.

5

6 

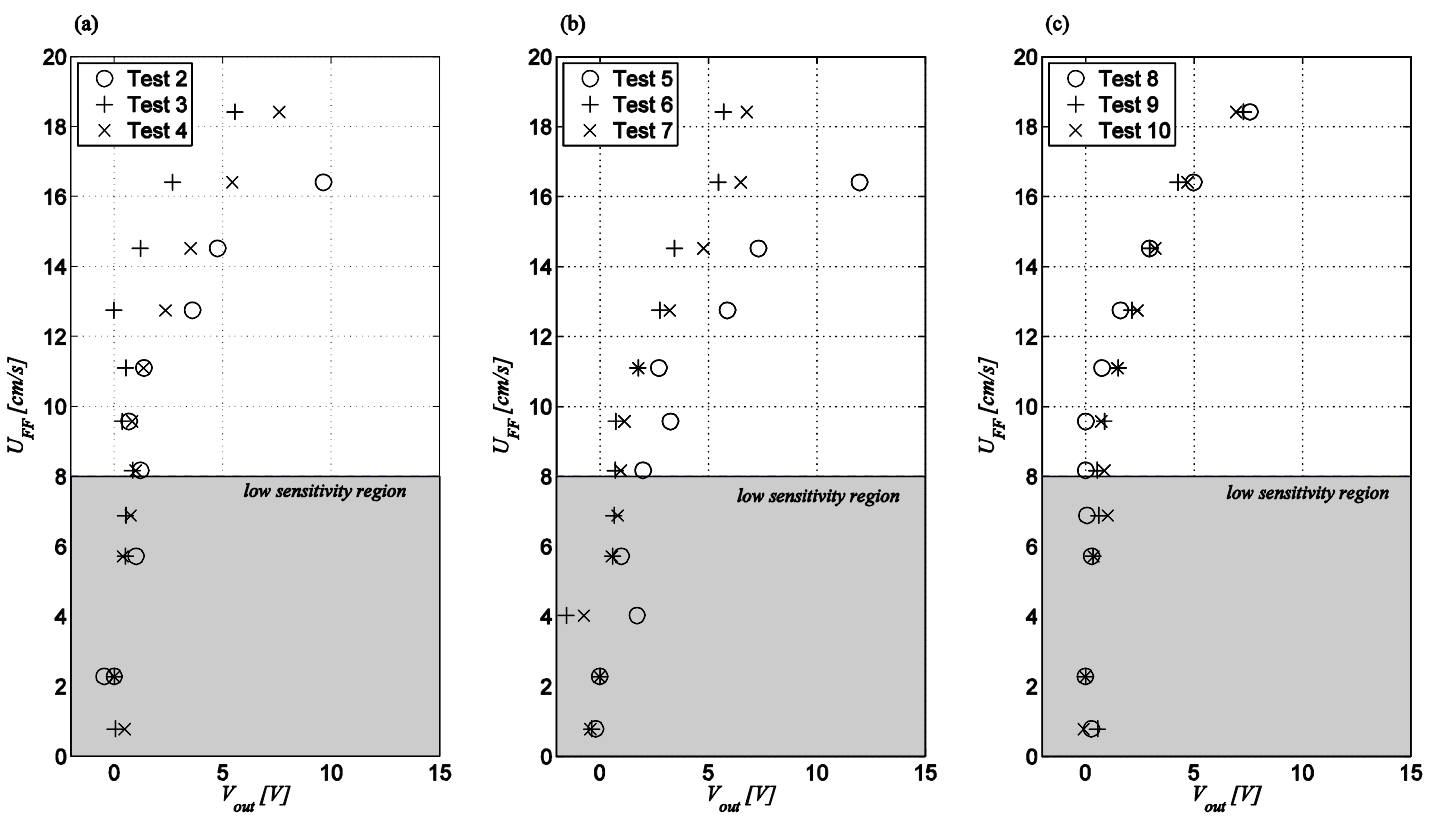

4 Figure 7 Relationship between the average output voltage $V_{\text {out }}$ and the velocity at the ferrofluid 5 location $U_{F F}$ for increasing values of external controlling magnetic force generated by : (a) no. 62 magnets S0805 type (Nominal force $\boldsymbol{F}=2 \cdot 19.61 \mathrm{~N}$ ); (b) no. 2 magnets S0808 type (Nominal 7 force $\boldsymbol{F}=2 \cdot 24.51 \mathrm{~N}$ ); (c) no. 3 magnets S0805 type (Nominal force $\boldsymbol{F}=3 \cdot 19.61 \mathrm{~N}$ ). The grey area 8 indicates a region within which the sensitivity of measuring system is very low, since the 9 ferrofluid spike is hardly deformed or moved by the action of the flow. 
(a)

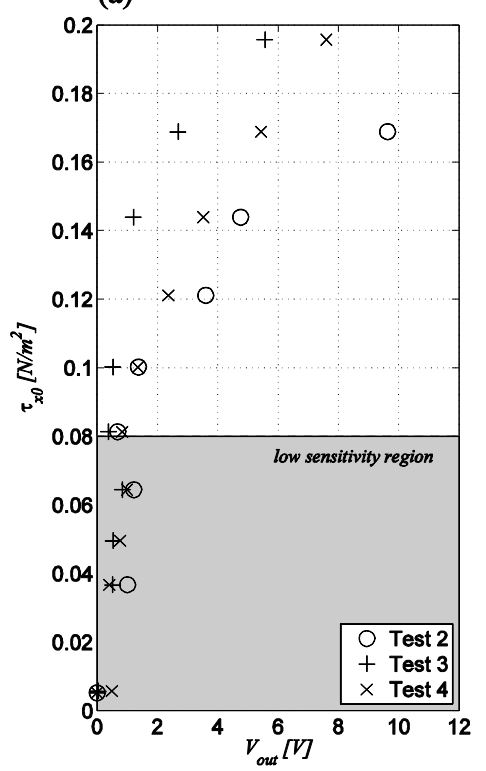

(b)

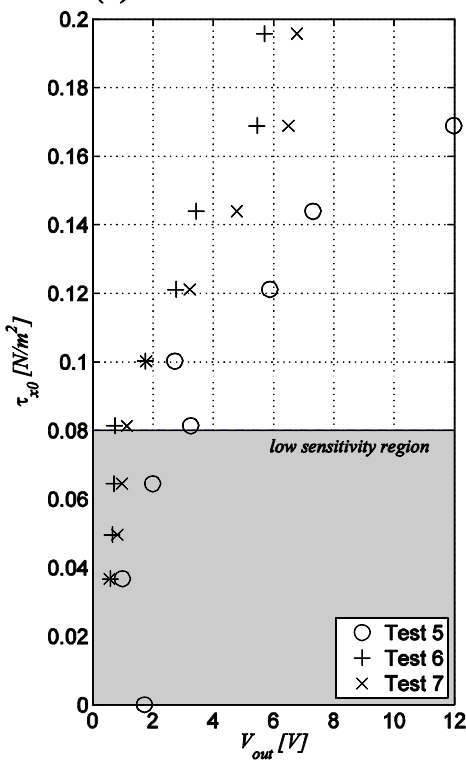

(c)

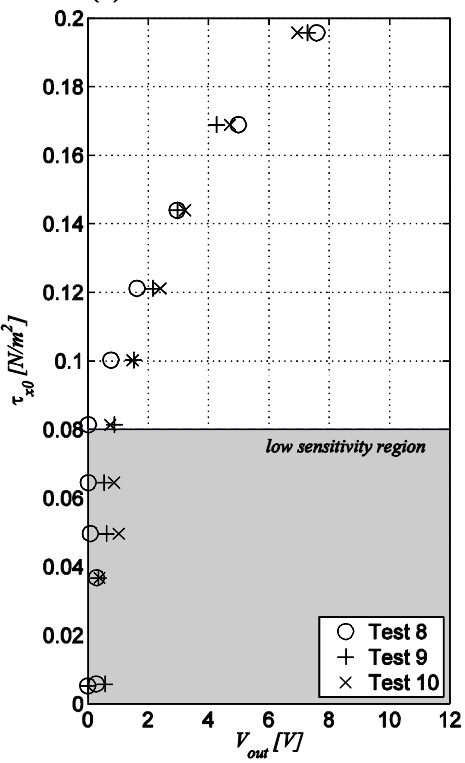

2

3 Figure 8 Relationship between the average output voltage $V_{\text {out }}$ and the bottom shear stress in

4 the flow direction $\tau_{x 0}$ for increasing values of external controlling magnetic force generated by 5 : (a) no. 2 magnets S0805 type (Nominal force $\boldsymbol{F}=2 \cdot 19.61 \mathrm{~N}$ ); (b) no. 2 magnets S0808 type 6 (Nominal force $\boldsymbol{F}=2 \cdot 24.51 \mathrm{~N}$ ); (c) no. 3 magnets S0805 type (Nominal force $\boldsymbol{F}=3 \cdot 19.61 \mathrm{~N}$ ). 

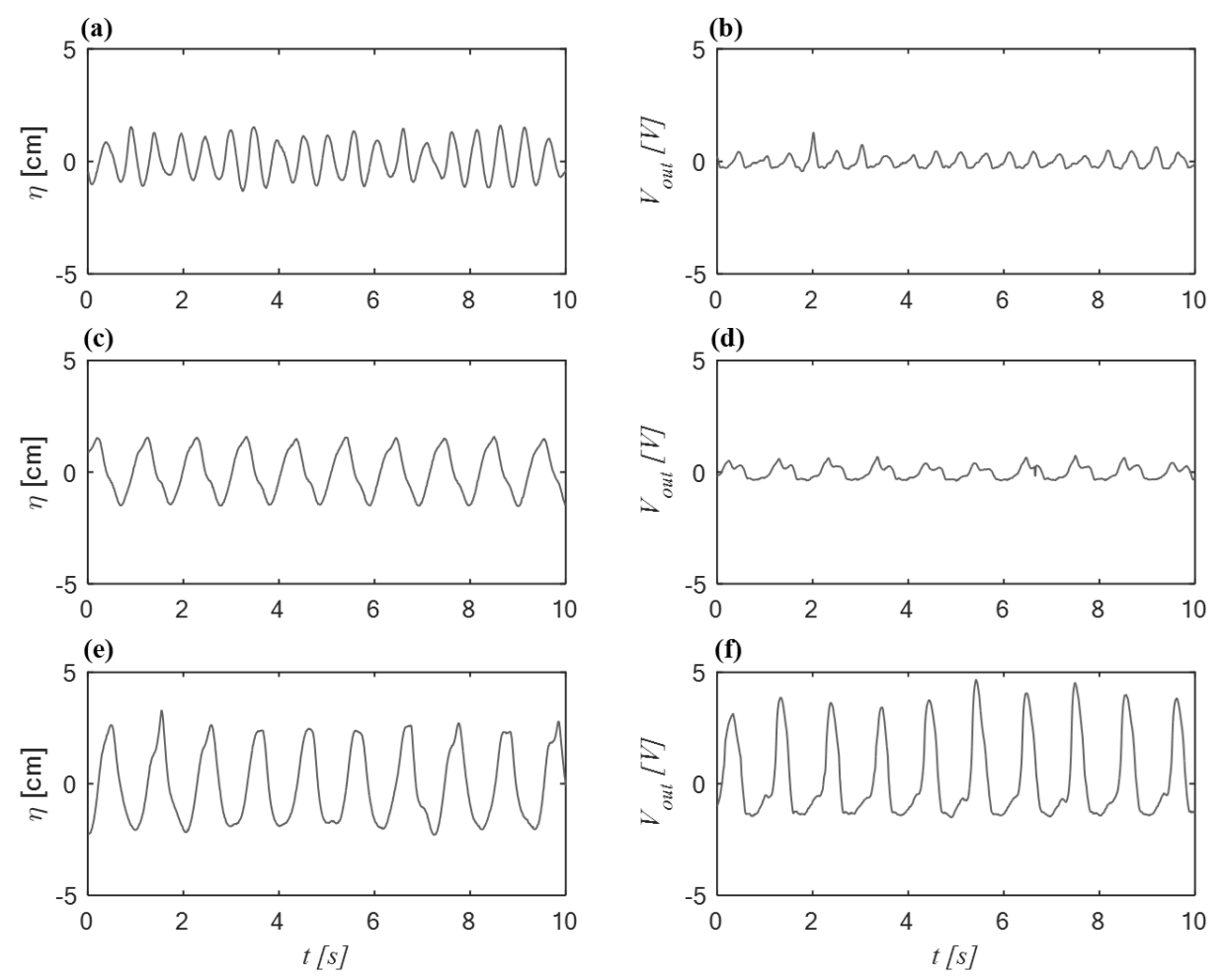

2

3 Figure 9 Examples of the time-dependent signals of the surface elevation measured by a wave 4 gauge (left column) and of the peak-to peak voltage output measured by the conditioning

5 circuit (right column). (a)-(b): $H=2.2 \mathrm{~cm}, T=0.51 \mathrm{~s}, d_{50}=0.56 \mathrm{~mm}$; (c)-(d): $H=4.0 \mathrm{~cm}, T=0.51$

$6 \mathrm{~s}, d_{50}=0.56 \mathrm{~mm}$; (e)-(f): ): $H=6.0 \mathrm{~cm}, T=1.03 \mathrm{~s}, d_{50}=0.56 \mathrm{~mm}$. 


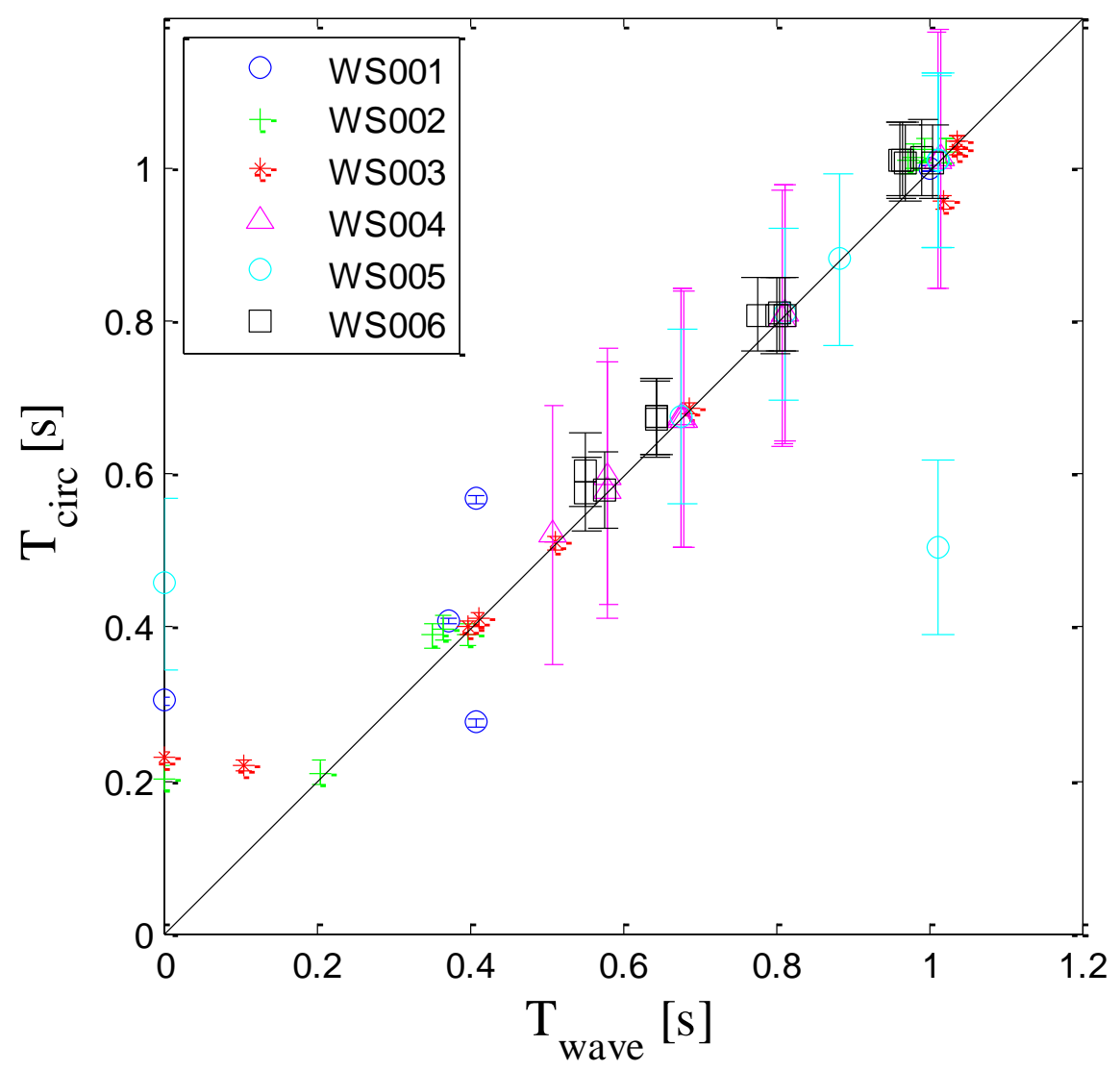

2

4 Figure 10 Wave periods measured independently by the conditioning circuit $T_{\text {circ }}$ and the 5 resistive wave gauges $T_{\text {wave }}$, by using different intensities of the external controlling magnetic 6 fields and different gains (see Table 5). The error bars show the standard deviation of the $T_{\text {circ }}$

7 Waves are in deep waters if the wave period is smaller than 0.51-0.62 s, depending on the 8 water depth value, which is in the range $h=0.25-0.30 \mathrm{~m}$. 
(a)

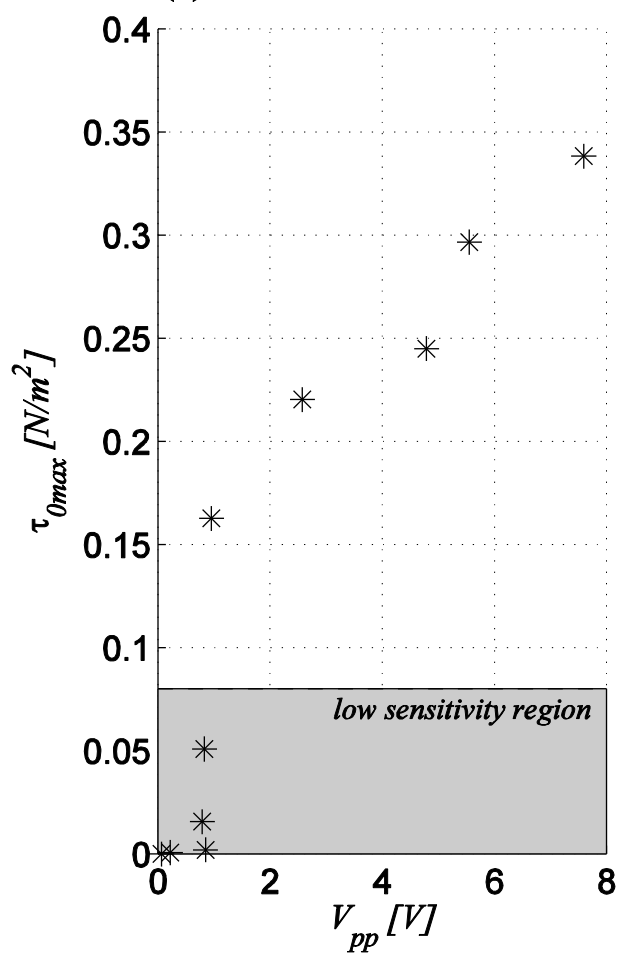

(b)

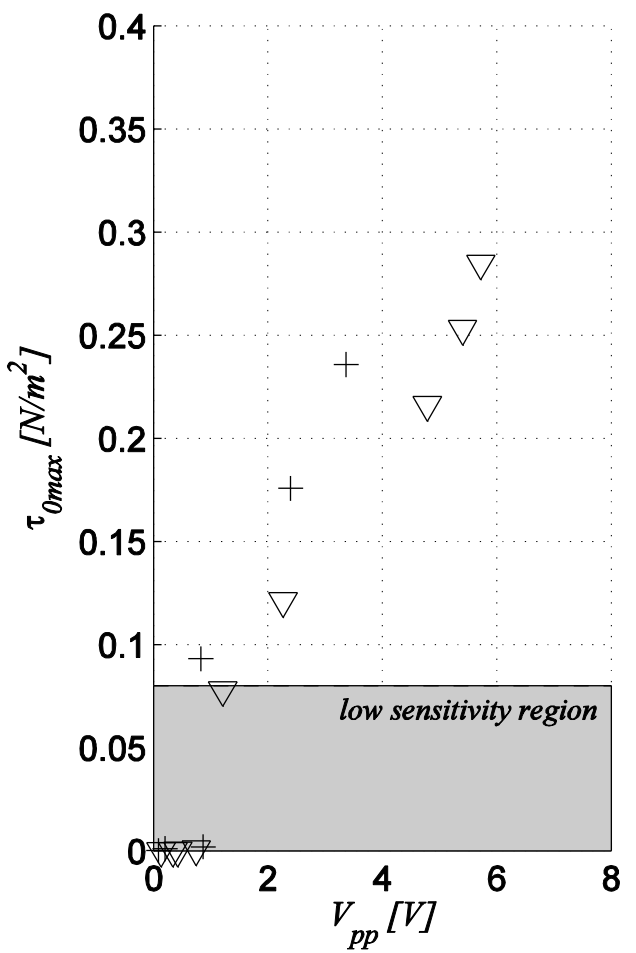

2

3 Figure 11 Relationship between the peak-to-peak output voltage of the conditioning circuit 4 and the maximum bed shear stress under surface regular waves.(a) WS003 (no. 2 magnets $5 \mathrm{~S} 0805, G=11.6, \tau_{c}=0.27 \mathrm{~N} / \mathrm{m}^{2}$ ); (b) WS001 (+) and WS002 ( $\left.\nabla\right)$ (no. 4 magnets S0805, $\left.6 G=11.6, \tau_{c}=0.27 \mathrm{~N} / \mathrm{m}^{2}\right)$. 
(a)

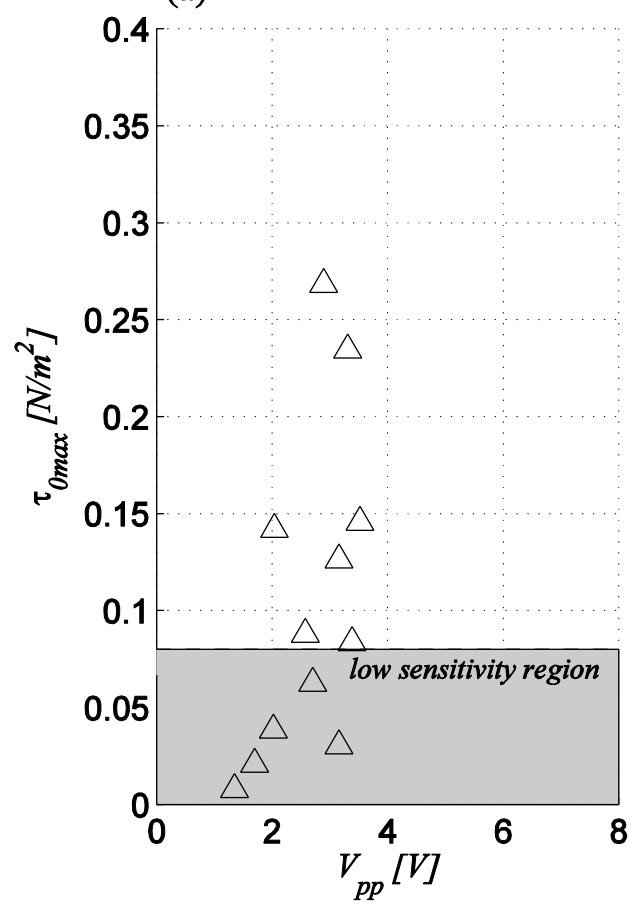

(b)

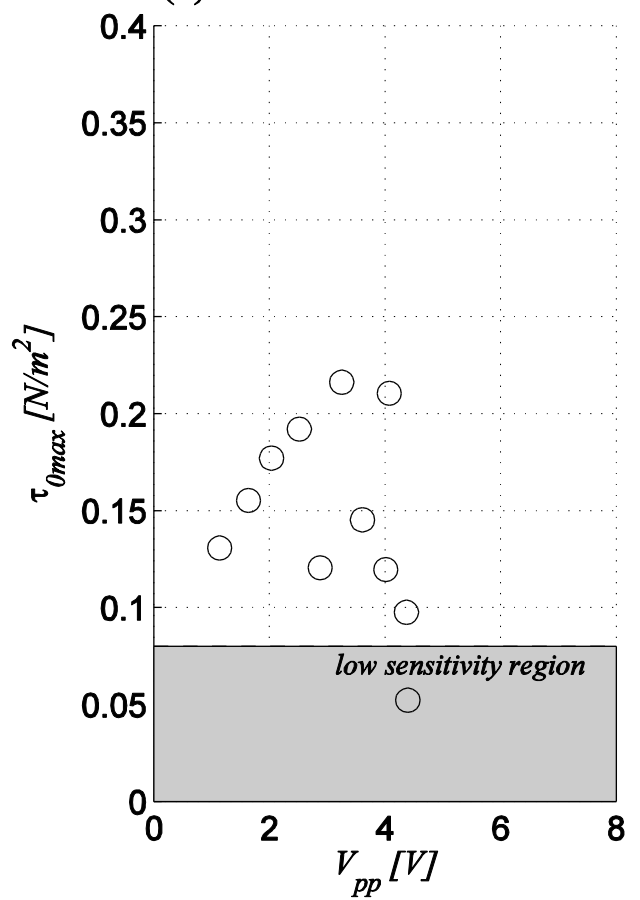

Figure 12 Relationship between the peak-to-peak output voltage of the conditioning circuit 4 and the maximum bed shear stress under surface regular waves.(a) WS004( no. 2 magnets 5 S0805, $G=25.0, \tau_{c}=0.17 \mathrm{~N} / \mathrm{m}^{2}$ ); (b)WS005 (no. 4 magnets S0805, $G=25.0, \tau_{c}=0.17 \mathrm{~N} / \mathrm{m}^{2}$ ). 
(a)

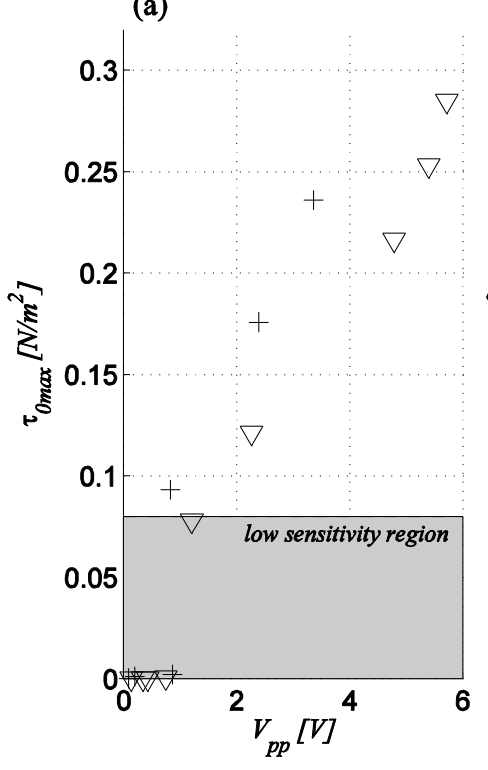

(b)

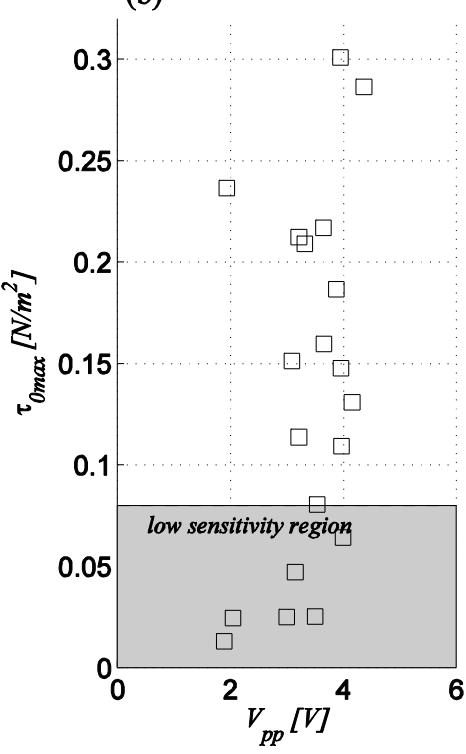

(c)

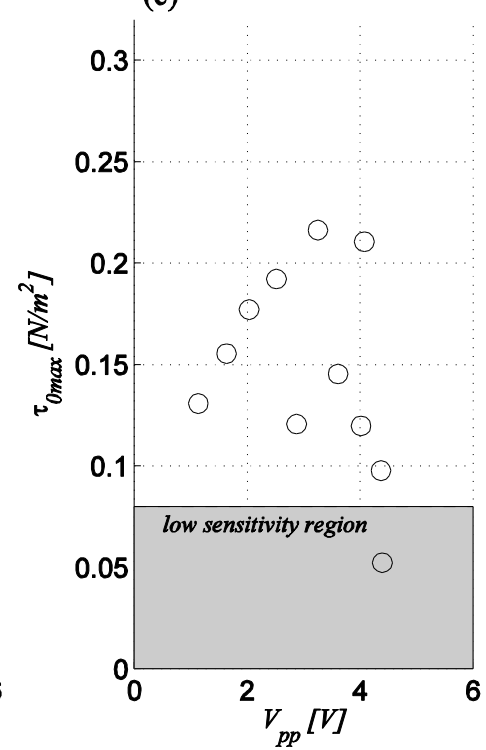

3 Figure 13 Relationship between the peak-to-peak output voltage of the conditioning circuit and 4 the maximum bed shear stress under surface regular waves.(a) WS001 (+) and WS002 ( $\nabla)$ (no. 54 magnets S0805, $G=11.6, \tau_{c}=0.27 \mathrm{~N} / \mathrm{m}^{2}$ ); (b) WS006 (no. 4 magnets $\mathrm{S} 0805, G=19.5, \tau_{c}=0.17$ $6 \mathrm{~N} / \mathrm{m}^{2}$ ); (c) WS005 (no. 4 magnets S0805, $G=25.0, \tau_{c}=0.17 \mathrm{~N} / \mathrm{m}^{2}$ ). 
(a)

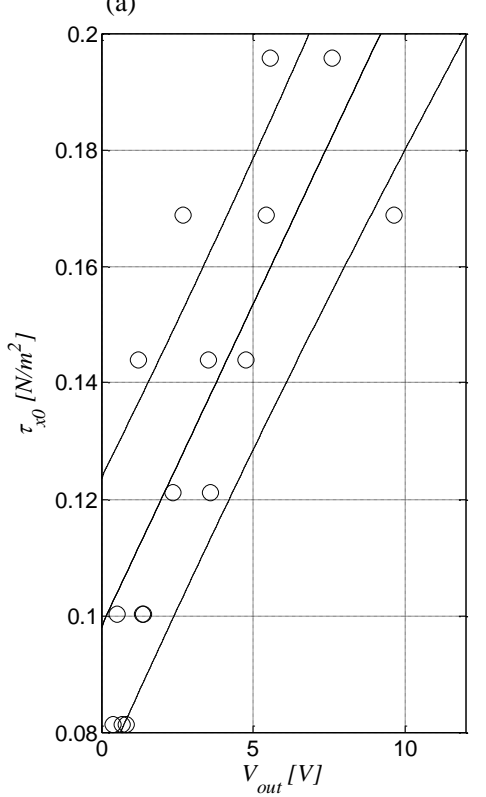

(b)

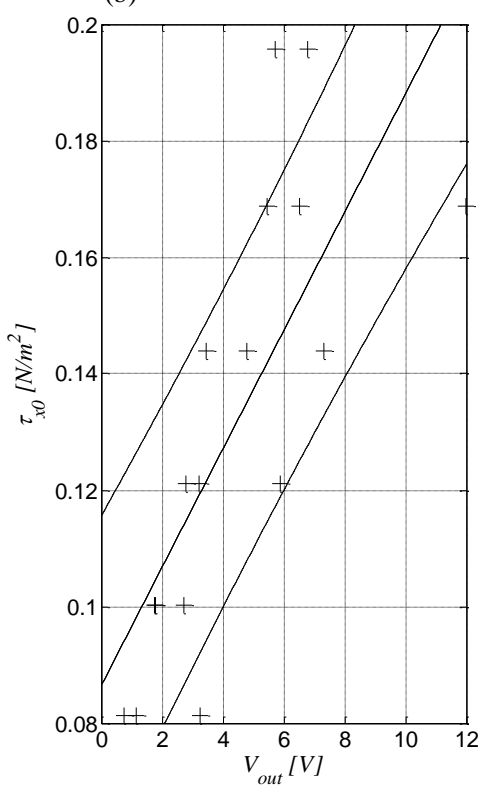

(c)

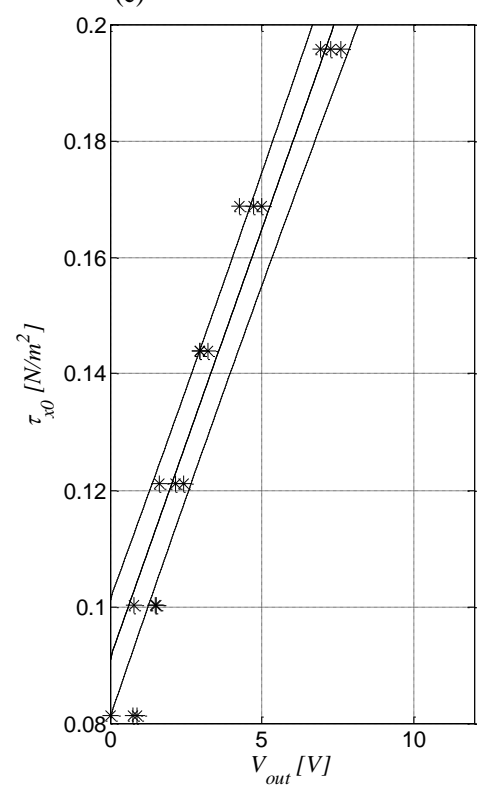

2 Figure 14 Steady current experiments: calibration and error estimates of the proposed ferrofluid 3 sensors of bottom shear stress $\tau_{x 0}$ for increasing values of external controlling magnetic force 4 generated by: (a) no. 2 magnets S0805 type (Nominal force $\boldsymbol{F}=2 \cdot 19.61 \mathrm{~N}$ ); (b) no. 2 magnets 5 S0808 type (Nominal force $\boldsymbol{F}=2 \cdot 24.51 \mathrm{~N}$ ); (c) no. 3 magnets S0805 type (Nominal force $6 \quad \boldsymbol{F}=3 \cdot 19.61 \mathrm{~N})$. The solid line represents the calibration curve, while the dashed lines indicate 7 the $50 \%$ confidence interval. 
(a)

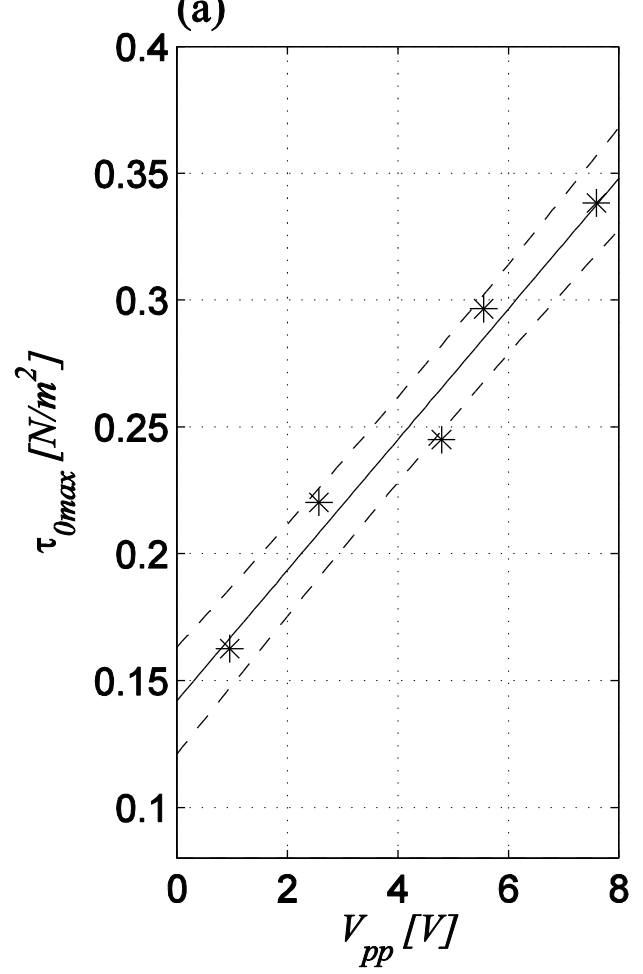

(b)

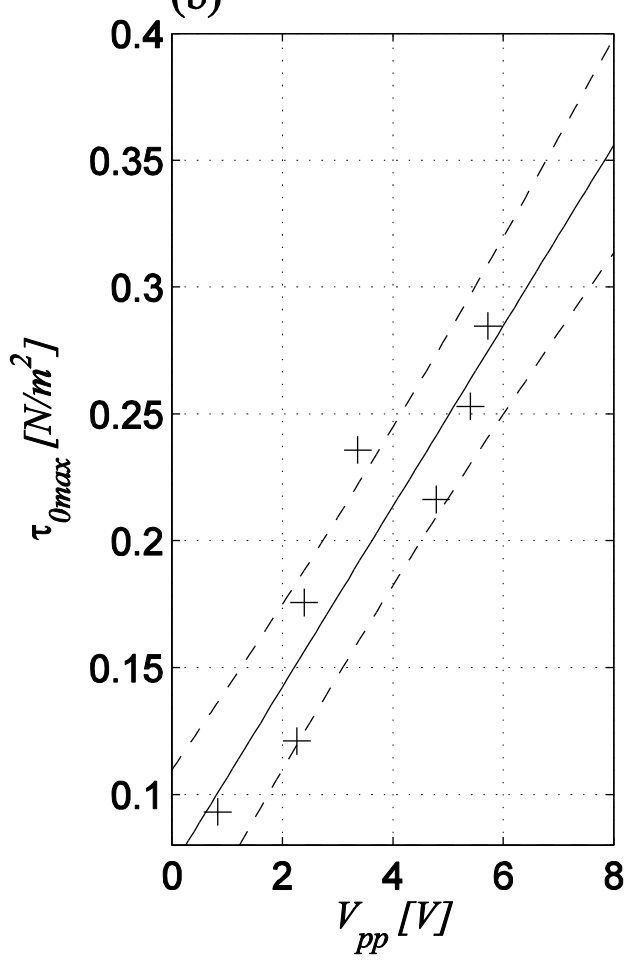

2 Figure 15 Monochromatic wave experiments: calibration and error estimates of the proposed

3 ferrofluid sensors of the maximum bed shear stress for different intensity of the external

4 controlling magnetic field generated by: (a) WS003: no. 2 magnets S0805 (Nominal force

$5 \boldsymbol{F}=2 \cdot 19.61 \mathrm{~N}$ ), $G=11.6, \tau_{c}=0.27 \mathrm{~N} / \mathrm{m}^{2}$; (b) WS001 no. 4 magnets S0805 (Nominal force

$6 \quad \boldsymbol{F}=4 \cdot 19.61 \mathrm{~N}), G=11.6, \tau_{c}=0.27 \mathrm{~N} / \mathrm{m}^{2}$. The solid line represents the calibration curve, while the

7 dashed lines indicate the 50\% confidence interval. In the tests, the critical bed stress for the

8 incipient motion of sediments is estimated to be about $0.27 \mathrm{~N} / \mathrm{m}^{2}$ for $d_{50}=0.56 \mathrm{~mm}$ and 0.17

$9 \mathrm{~N} / \mathrm{m}^{2}$ for $d_{50}=0.24 \mathrm{~mm}$. 